Hochschule Aalen

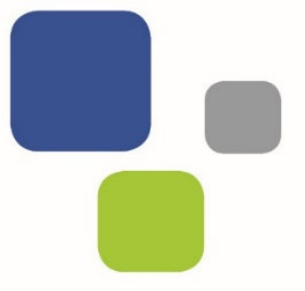

AAUF

Aalener Institut für Unternehmensführung

Wertorientierung - Governance

\title{
WERTORIENTIERUNG GOVERNANCE
}

\section{FAMILY BUSINESS \\ VERANTWORTUNG INTEGRITÄT}

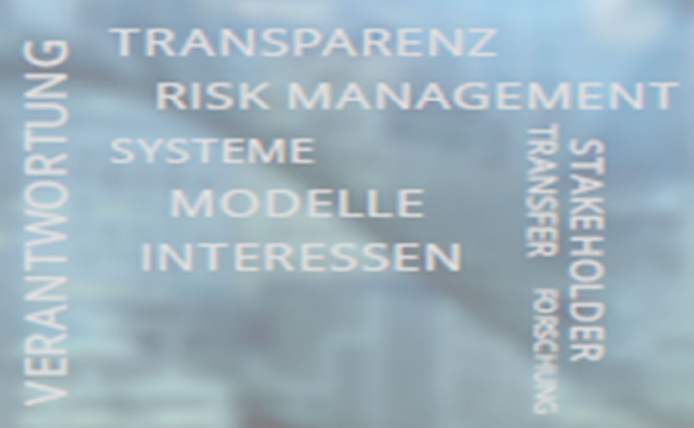

Hygiene-Compliance im Krankenhaus

Studienserie „Erfolgsfaktoren der Unternehmensführung"

Band 6, ISBN 978-3-947393-05-3 


\title{
Impressum
}

\author{
Direktorium Professor Dr. habil. Patrick Ulrich \\ Professor Dr. Ingo Scheuermann \\ Wissenschaftlicher Projektleiter Professor Dr. habil. Patrick Ulrich \\ Weitere beteiligte Personen Mona Kratt \\ Herausgeber Professor Dr. habil. Patrick Ulrich \\ Professor Dr. Ingo Scheuermann \\ Hochschule Aalen \\ Aalener Institut für Unternehmensführung (AAUF) \\ Beethovenstr. 1 \\ D-73430 Aalen \\ Copyright @ @ 2020 by Aalener Institut für Unternehmensführung (AAUF) \\ Druck Aalen 2020 \\ Printed in Germany
}




\section{Inhaltsverzeichnis}

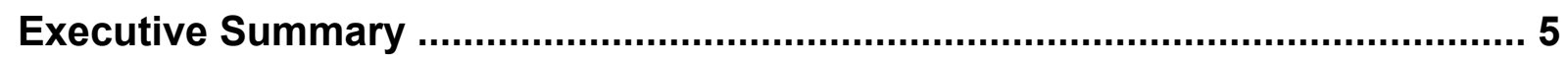

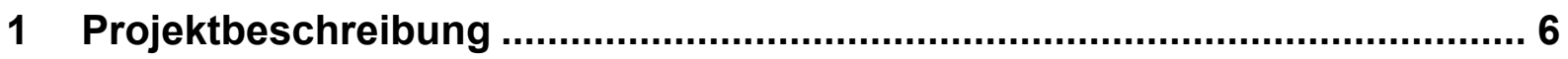

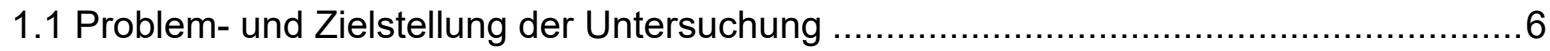

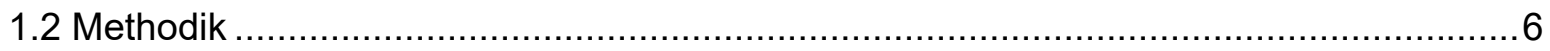

2 Angaben zum Krankenhaus .............................................................. 7

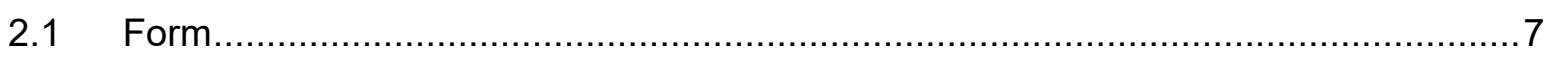

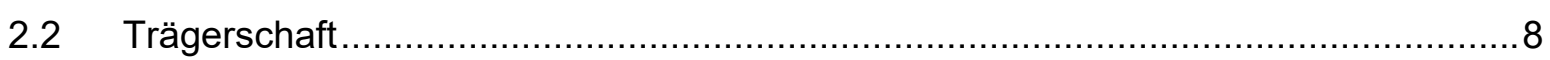

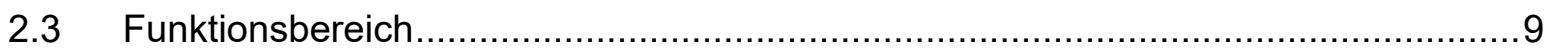

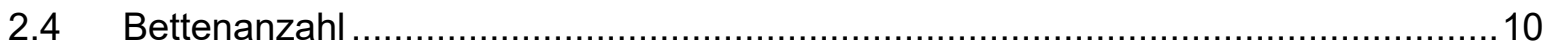

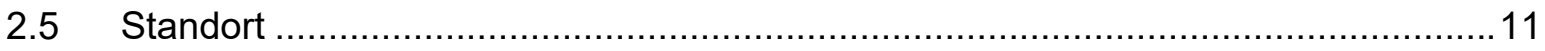

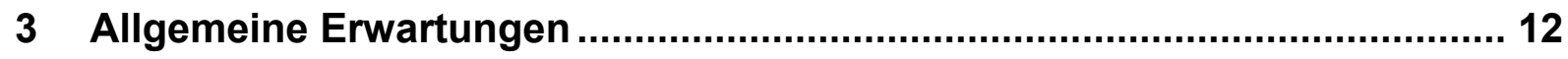

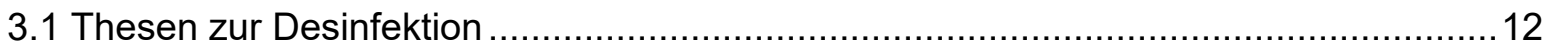

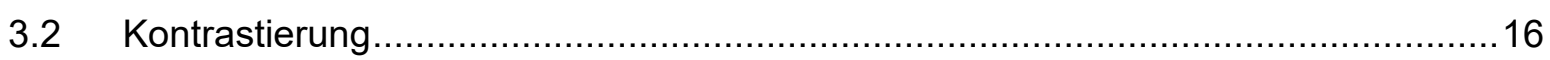

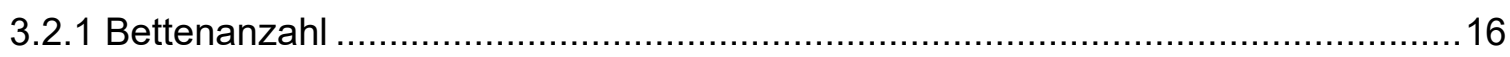

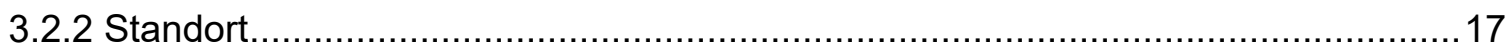

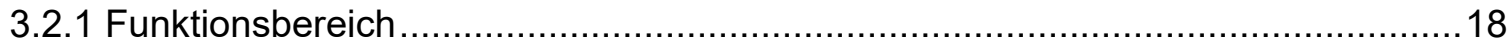

$4 \quad$ Fragen zur Desinfektion ..................................................................... 19

4.1 Einhaltungsgrad der Regeln zur Händedesinfektion............................................19

4.2 Rate nosokomialer Infektionen im Krankenhaus ...........................................20

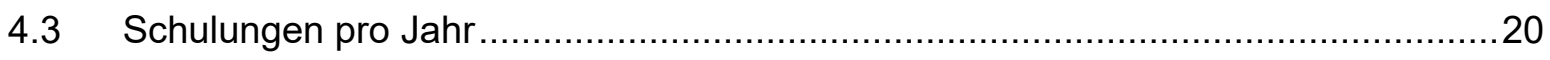

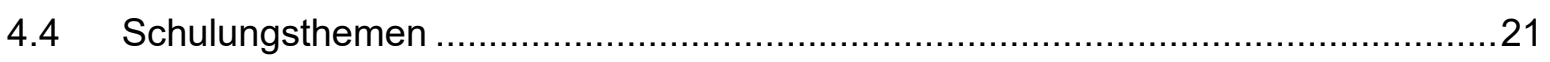

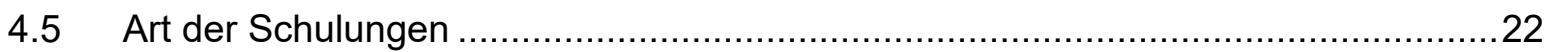

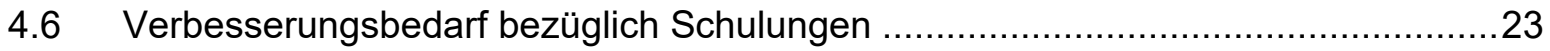

$4.7 \quad$ Interesse an begleitenden Schulungen durch die Industrie ...............................24

4.8 Einschätzung der Kapazitäten für Observationen/Beobachtungen der

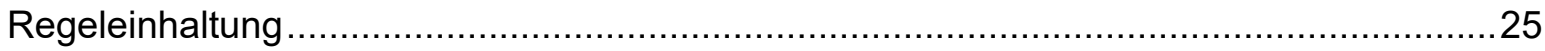

4.9 Akzeptanz von Pflegekräften im Vergleich zu ärztlichem Personal .......................26

4.12 Einschätzung des Kommunikationsniveaus im Krankenhaus................................29

4.13 Compliance-Quote im Bereich Flächendesinfektion ............................................ 30

4.14 Drei Aufgabenträger, die Flächendesinfektion durchführen...................................31

4.15 Bewertung von Aussagen zu Feedback zum Krankenhaus ................................ 32

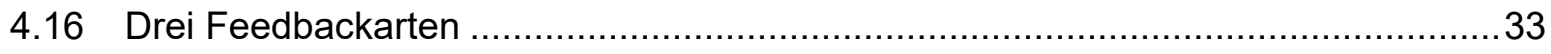

4.17 Einschätzung der Datensituation als Grundlage für ein Feedback ........................34

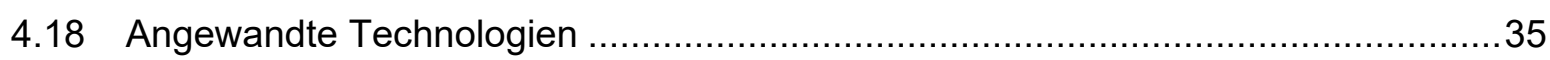

4.19 Unterstützung von Technologien in konkreten Aspekten .....................................36 


\section{Abbilldungsverzeichnis}

Abbildung 1: Form der untersuchten Krankenhäuser .............................................................. 7

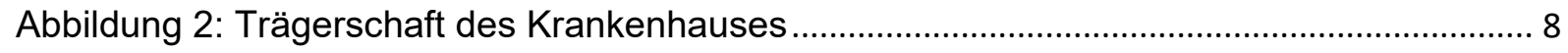

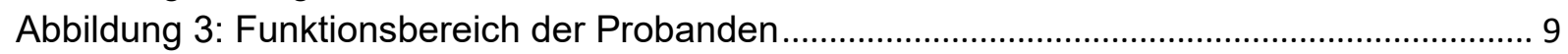

Abbildung 4: Bettenanzahl der befragten Krankenhäuser ....................................................... 10

Abbildung 5: Standort der befragten Krankenhäuser............................................................. 11

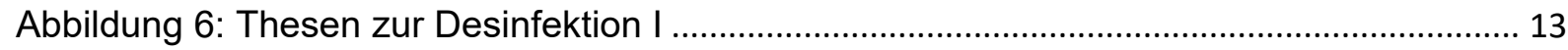

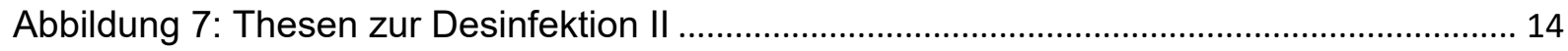

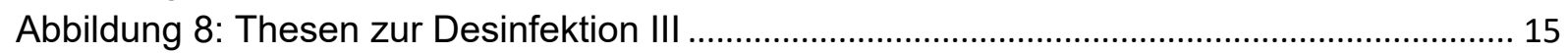

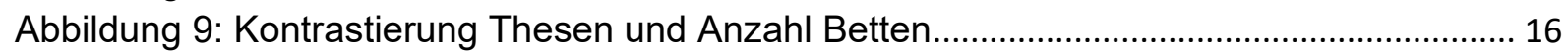

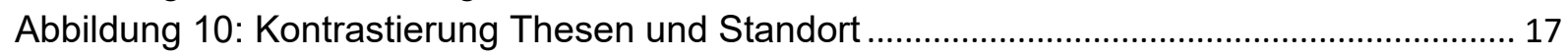

Abbildung 11: Kontrastierung Thesen und Funktionsbereich ................................................. 18

Abbildung 12: Einschätzung des Einhaltungsgrades der Regeln .......................................... 19

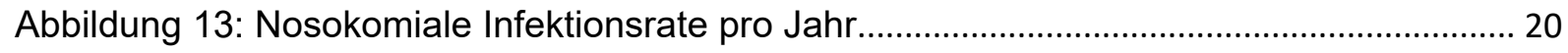

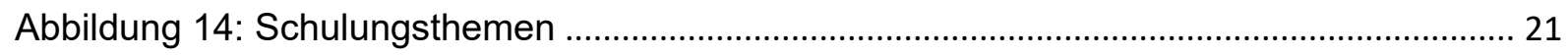

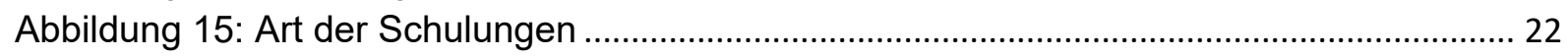

Abbildung 16: Verbesserungsbedarf bezüglich Schulungen ................................................. 23

Abbildung 17: Interesse der Krankenhäuser an begleitenden Schulungen durch die Industrie

Abbildung 18: Einschätzung der Kapazitäten für Observationen............................................... 25

Abbildung 19: Akzeptanz von Pflegekräften im Vergleich zu ärztlichem Personal .................. 26

Abbildung 20: Einschätzung von Hygienefachkräften und -teams .......................................... 27

Abbildung 21: Gründe für die Nicht-Einhaltung der WHO-Regeln .......................................... 28

Abbildung 22: Einschätzung des Kommunikationsniveaus...................................................... 29

Abbildung 23: Einschätzung der Compliance-Quote ............................................................. 30

Abbildung 24: Aufgabenträger der Flächendesinfektion ...................................................... 31

Abbildung 25: Aussagen zu Feedback im Krankenhaus....................................................... 32

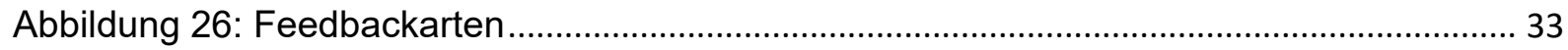

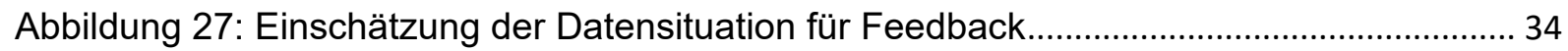

Abbildung 28: Technologien, die Compliance unterstützen..................................................... 35

Abbildung 29: Bereiche, in denen Technologien unterstützen können ..................................... 36 


\section{Executive Summary}

Die vorliegende Studie beschäftigt sich mit der Hygiene-Compliance in Krankenhäusern. Zur Einhaltung von Regeln der Hände- und Flächendesinfektion wurden hierzu Entscheider von Krankenhäusern in Deutschland, Österreich und der Schweiz befragt. In der Studie zeigt sich eine starke Sensibilisierung für das Thema Compliance. Hier ist hinzuzufügen, dass die Studie vor der Corona-Pandemie durchgeführt wurde, und bereits zu diesem Zeitpunkt gab es eine Aufmerksamkeit für das Thema.

Die Ergebnisse der Studie zeigen, dass die Entscheider im Bereich Hände- und Flächendesinfektion bei den Mitarbeiterinnen und Mitarbeitern noch Nachholbedarf sehen, da nicht alle für die Thematik sensibilisiert sind. Als mögliche Lösungsalternativen werden neben Schulungsmaßnahmen auch digitale Technologien wie App-Lösungen diskutiert.

Interessant ist auch die individuelle Untersuchung der Gründe für die Nicht-Einhaltung der Regeln. Stress, Zeitmangel und personelle Unterversorgung werden hier von den jeweiligen Personen als häufigste Rechtfertigungsgründe für die Nicht-Einhaltung der WHO-Regeln genannt. 


\section{Projektbeschreibung}

\subsection{Problem- und Zielstellung der Untersuchung}

Nosokomiale Infektionen sind in Krankenhäusern stets ein präsentes Thema. Die Ursachen liegen in der Nicht-Einhaltung vorgegebener Hygienestandards zur Händeund Flächendesinfektion. Faktoren, wie Stress, Unkenntnis, oder die Schädlichkeit für die Hände spielen dabei eine zentrale Rolle.

Das Hygiene-Compliance ist für die Einhaltung der Regelungen zuständig. Herausforderungen bestehen vor allem in der Überprüfung und Prophylaxe der Regelverstöße, was sich durch (digitale) Technologien vereinfachen lässt.

Das Ziel der Erhebung ist die Abfrage des aktuellen Stands der Hygiene-Compliance in Krankenhäusern, sowie die Ermittlung von Möglichkeiten der Unterstützung, um das Infektionsrisiko langfristig zu senken.

\subsection{Methodik}

Die Datenerhebung erfolgte mit Hilfe eines standardisierten Online-Fragebogens, der offene und geschlossene Fragen enthielt. Die Befragung erfolgte im Zeitraum vom 19.11.2019 bis zum 31.12.2019. Hierfür wurden vorab mit Hilfe von Datenbanken per Zufallsprinzip E-Mail-Adressen von Krankenhäusern aus Deutschland, Österreich und der Schweiz generiert.

Insgesamt wurden 1.077 Krankenhäuser per E-Mail kontaktiert. Der Onlinefragebogen wurde im Befragungszeitraum 162-mal aufgerufen, was einer Teilnahmequote von 15 Prozent entspricht. 151 Befragte beantworteten die gestellten Fragen, wobei 34 Krankenhäuser die Umfrage vorzeitig abgebrochen haben.

In diesem Zusammenhang ist anzumerken, dass es bei einzelnen Fragegestellungen dennoch zu unterschiedlichen Nennungen kommen kann, da der partielle Antwortausfall (Item-Non-Response) im vorliegenden Ergebnisbericht nicht berücksichtigt wurde. Dies liegt daran, dass bei der Gestaltung des Fragebogens bewusst auf das Festlegen von Pflichtfragen verzichtet wurde, da teilweise sehr themenspezifische und sensible Daten abgefragt wurden. Die Auswertung der Daten erfolgte mittels Microsoft Excel. 


\section{Angaben zum Krankenhaus}

\subsection{Form}

Die Umfrage zeigt, dass es sich bei mehr als der Hälfte (52 Prozent) der befragten Krankenhäuser um Allgemeinkliniken handelt. Bei 20 Prozent handelt es sich um Fachkliniken und bei weiteren 20 Prozent um Universitätskliniken. 7 Prozent sind Belegkrankenhäuser und ein Prozent um Praxiskliniken.

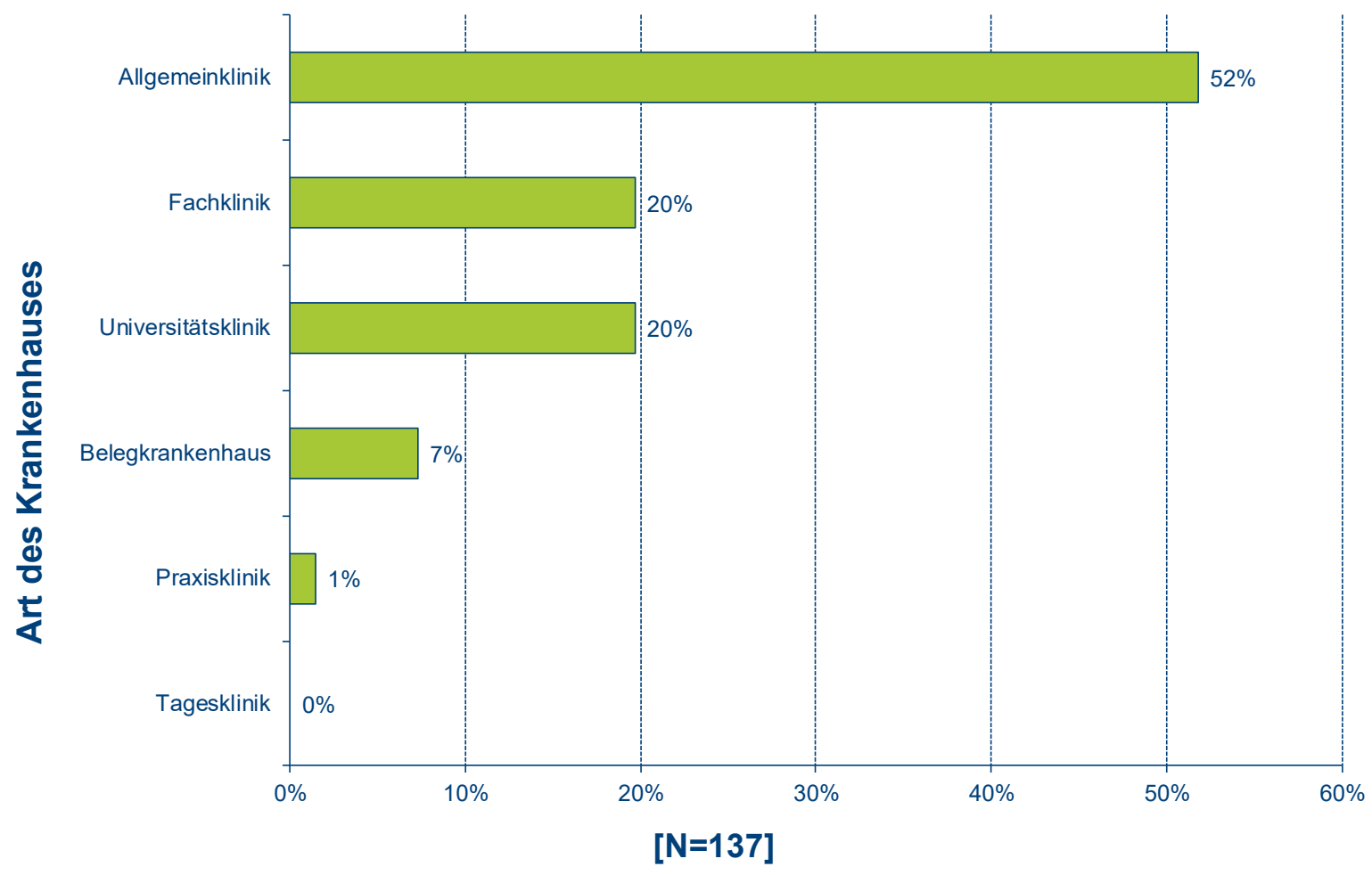

Abbildung 1: Form der untersuchten Krankenhäuser 


\subsection{Trägerschaft}

Um herauszufinden, ob es sich um eine öffentliche oder private Klinik handelt, wurden weiter sämtliche Teilnehmer befragt. Die Erhebung zeigt: Es handelt sich überwiegend um öffentliche Kliniken. 71 Prozent der Kliniken sind öffentliche Kliniken, wohingegen 29 Prozent privat organisiert sind.

\section{Öffentlich oder Privat}

[N=139]

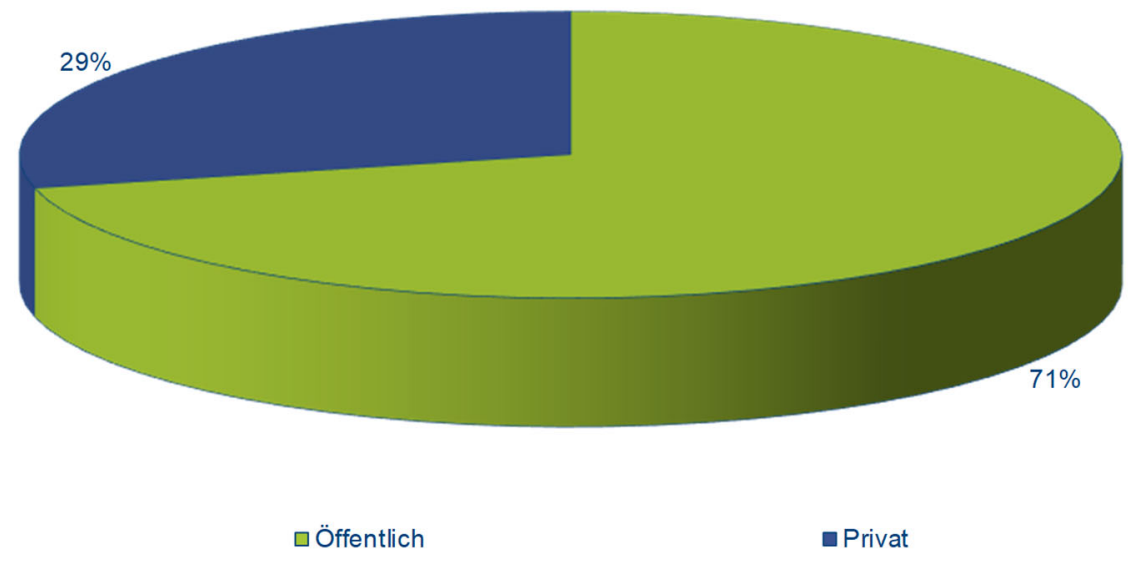

Abbildung 2: Trägerschaft des Krankenhauses 


\subsection{Funktionsbereich}

Über die Hälfte der Befragten (56 Prozent) sind in der Krankenhaushygiene-Tätig. 14 Prozent sind in der Pflege und 8 Prozent im Krankenhaus-Management tätig. Weitere 8 Prozent sind im Funktionsbereich leitendes ärztliches Personal und 7 Prozent im Funktionsbereich ärztliches Personal. Ein kleiner Anteil der Befragten ist im Pflegemanagement (6 Prozent), im Flächenmanagement (1 Prozent) und im Facility Management (1 Prozent) tätig.

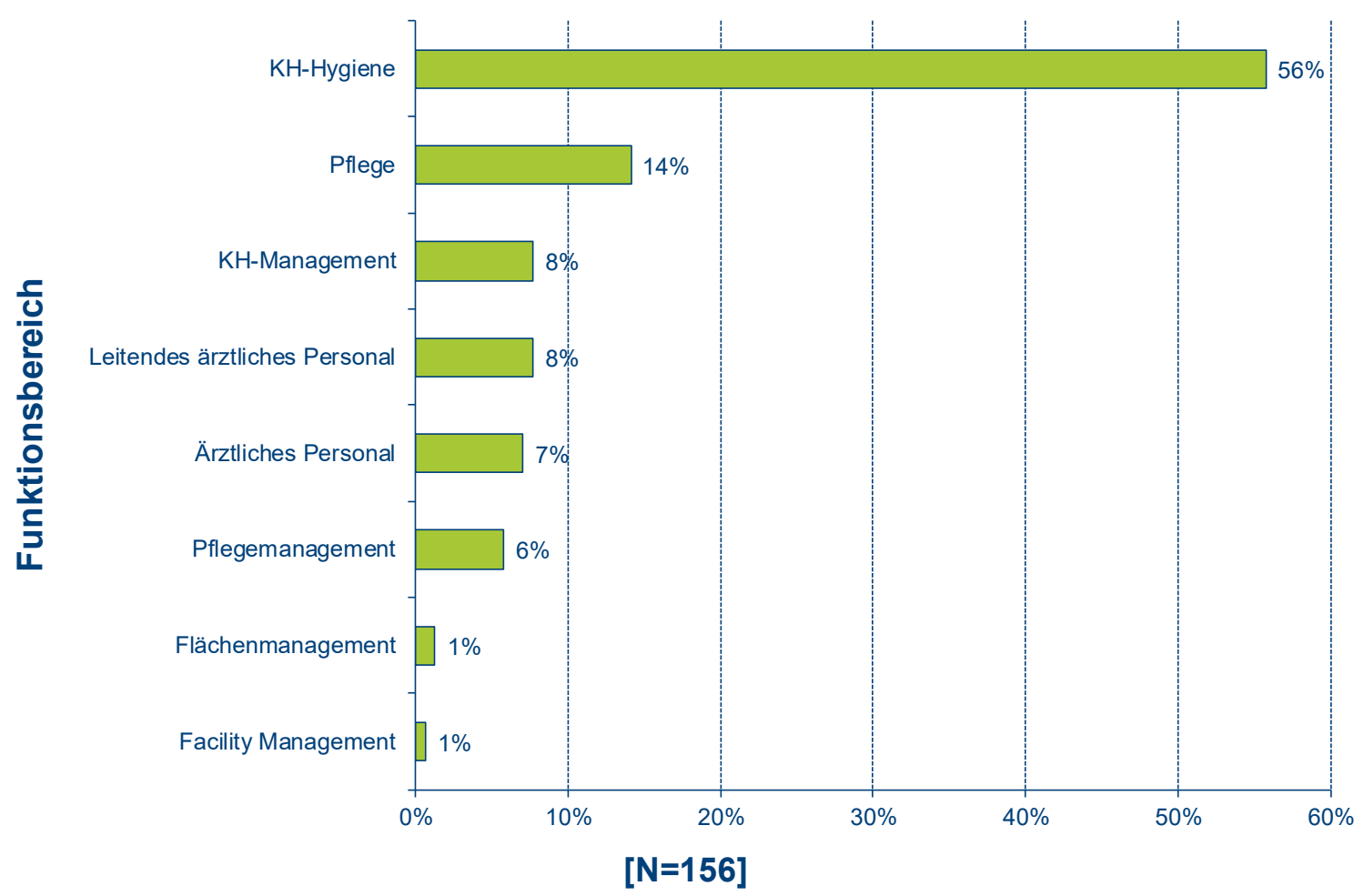

Abbildung 3: Funktionsbereich der Probanden 


\subsection{Bettenanzahl}

Etwas über die Hälfte der Krankenhäuser (54 Prozent) stehen zwischen 500 und 1000 Betten zur Verfügung. 20 Prozent der befragten Krankenhäuser geben an, dass Ihnen über 1000 Betten zur Verfügung stehen. 14 Prozent der Krankenhäuser teilen mit, dass Sie zwischen 100 und 500 und 11 Prozent zwischen 50 und 100 Betten haben. Nur 2 Prozent der befragten Krankenhäuser geben an, dass Ihnen weniger als 50 Betten zur Verfügung stehen.

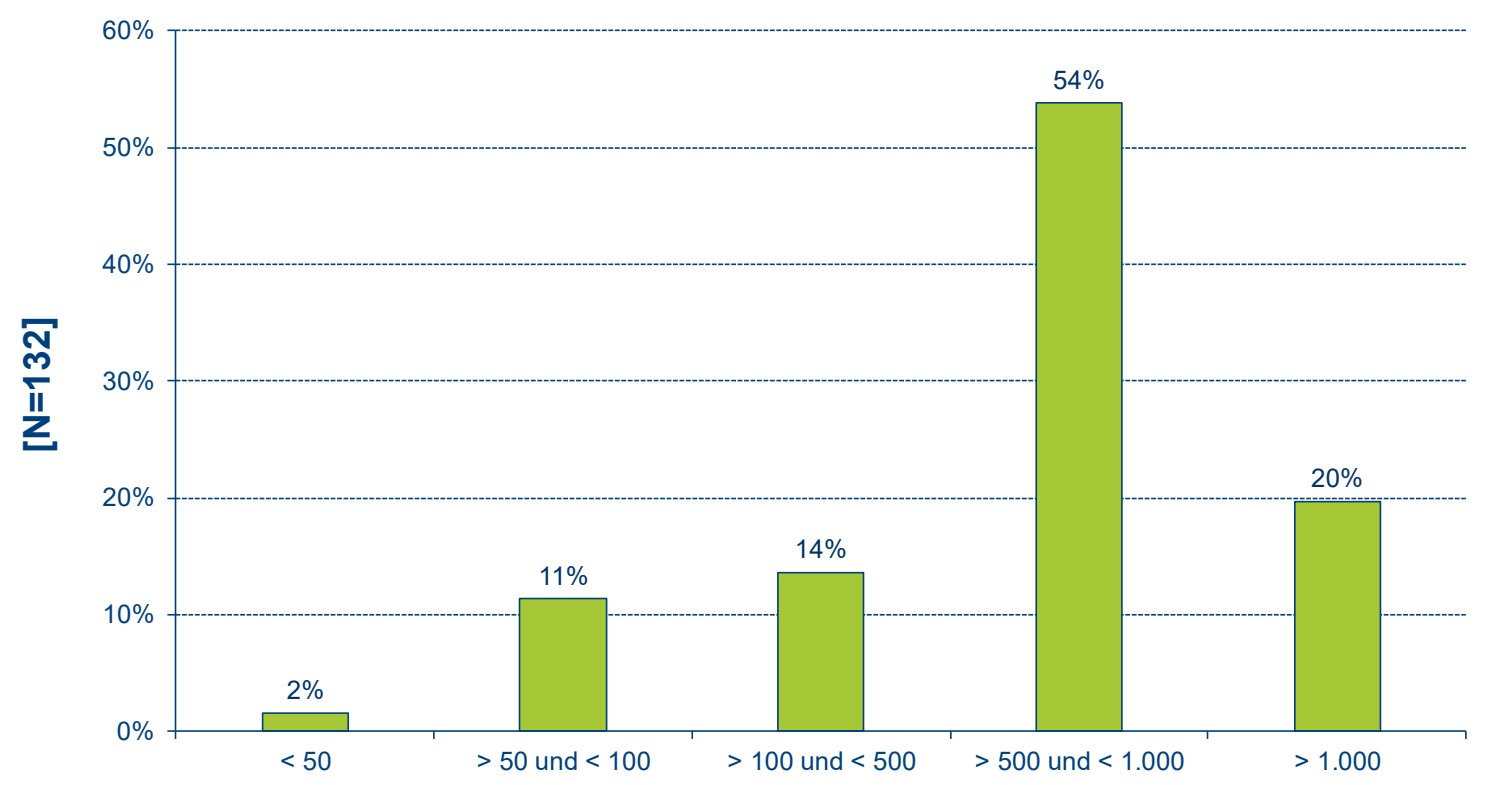

Anzahl der Betten

Abbildung 4: Bettenanzahl der befragten Krankenhäuser 


\subsection{Standort}

Die Herkunft der befragten Krankenhäuser verteilt sich relativ gleichmäßig. Mit 35 Prozent handelt es sich bei den befragten Krankenhäusern um Kliniken deutscher Herkunft. Dicht gefolgt von 34 Prozent der Krankenhäuser, die sich in Österreich und 31 Prozent, die sich in der Schweiz befinden.

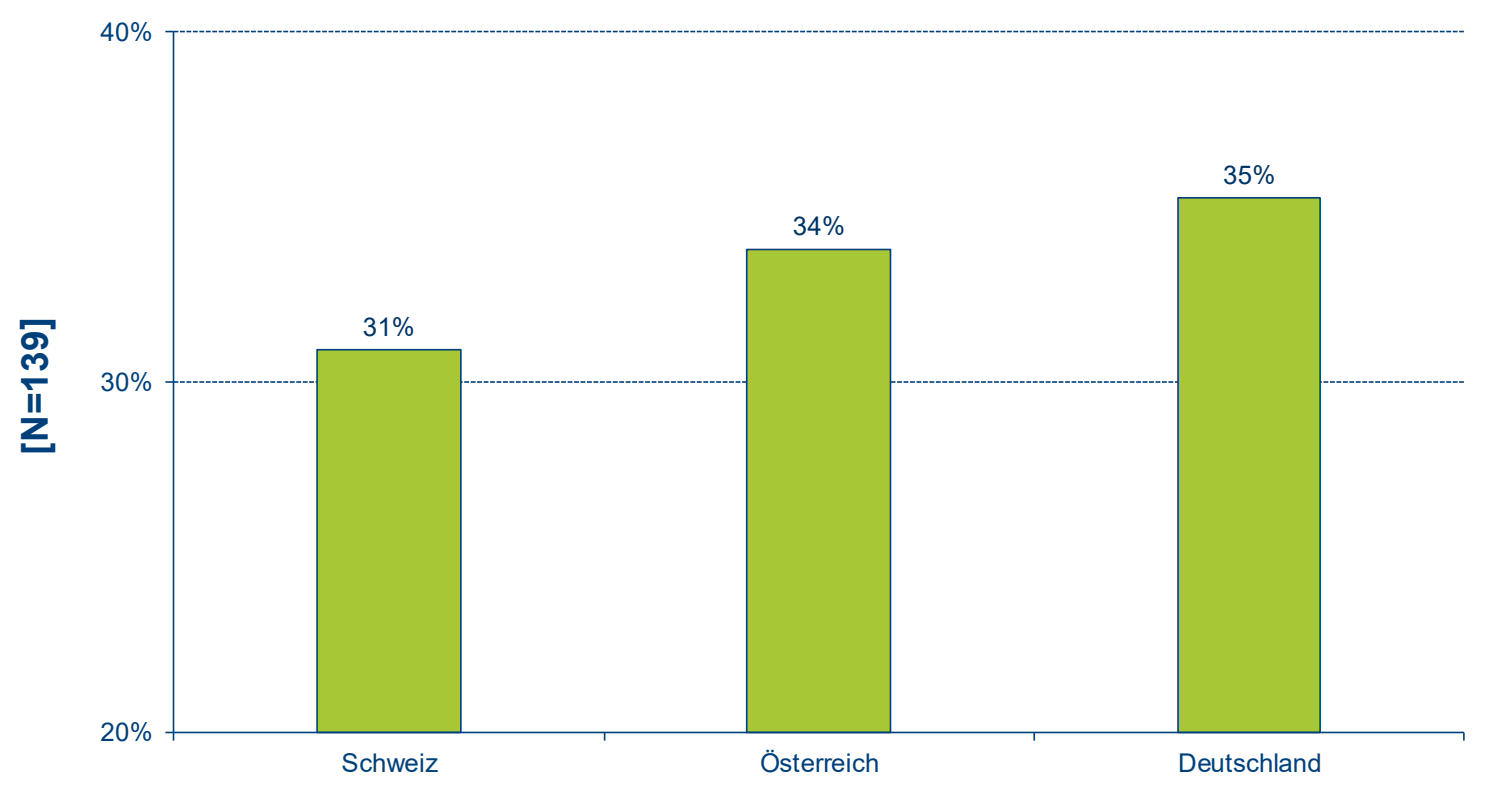

\section{Land des Krankenhauses}

\section{Abbildung 5: Standort der befragten Krankenhäuser}




\section{Allgemeine Erwartungen}

\subsection{Thesen zur Desinfektion}

Im Folgenden werden die Thesen zur Desinfektion formuliert. Dabei wird angenommen, dass die These ab einer Zustimmung (Summe aus sehr stark und stark) von 70 Prozent als bestätigt gilt. Bei einer Zustimmung von 30 - 70 Prozent wird die These teilweise bestätigt und bei unter 30 Prozent verworfen.

1. Händehygiene Compliance hat einen maßgeblichen Einfluss auf die Infektionsrate im jeweiligen Krankenhaus.

$\rightarrow$ These wird bestätigt: 98 Prozent der Probanden (63 Prozent sehr stark, 35 Prozent stark) sind der Meinung, dass Händehygiene Compliance einen maßgeblichen Einfluss auf die Infektionsrate hat.

2. Direktes Feedback ist der effektivste Weg, den Prozess der Verhaltensänderung mit den Betroffenen anzustoßen.

$\rightarrow$ These wird bestätigt: 92 Prozent (44 Prozent sehr stark, 48 Prozent stark) sind der Meinung, dass Feedback der effektivste Weg ist, den Prozess der Verhaltensänderung anzustoßen.

3. Unterstützung durch zuverlässige (digitale) Technologien ist akzeptabel und gewünscht, wenn sie den Betroffenen dient in dem sie Transparenz herstellt und allen hilft, bessere Abläufe zu gestalten.

$\rightarrow$ These wird bestätigt: 80 Prozent (31 Prozent sehr stark, 49 Prozent stark) empfinden Technologien als hilfreich, sofern sie Transparenz herstellen und bei der Gestaltung besserer Abläufe unterstützen.

4. Trotz ausreichender Schulungen wird die Händehygiene Compliance nicht ausreichend eingehalten - eine Verhaltensänderung ist schwer einzuleiten.

$\rightarrow$ These wird bestätigt: Insgesamt 74 Prozent der Probanden (20 Prozent sehr stark, 54 Prozent stark) bestätigen, dass die Compliance trotz Schulungen nicht eingehalten wird und eine Verhaltensänderung schwer einzuleiten ist. 


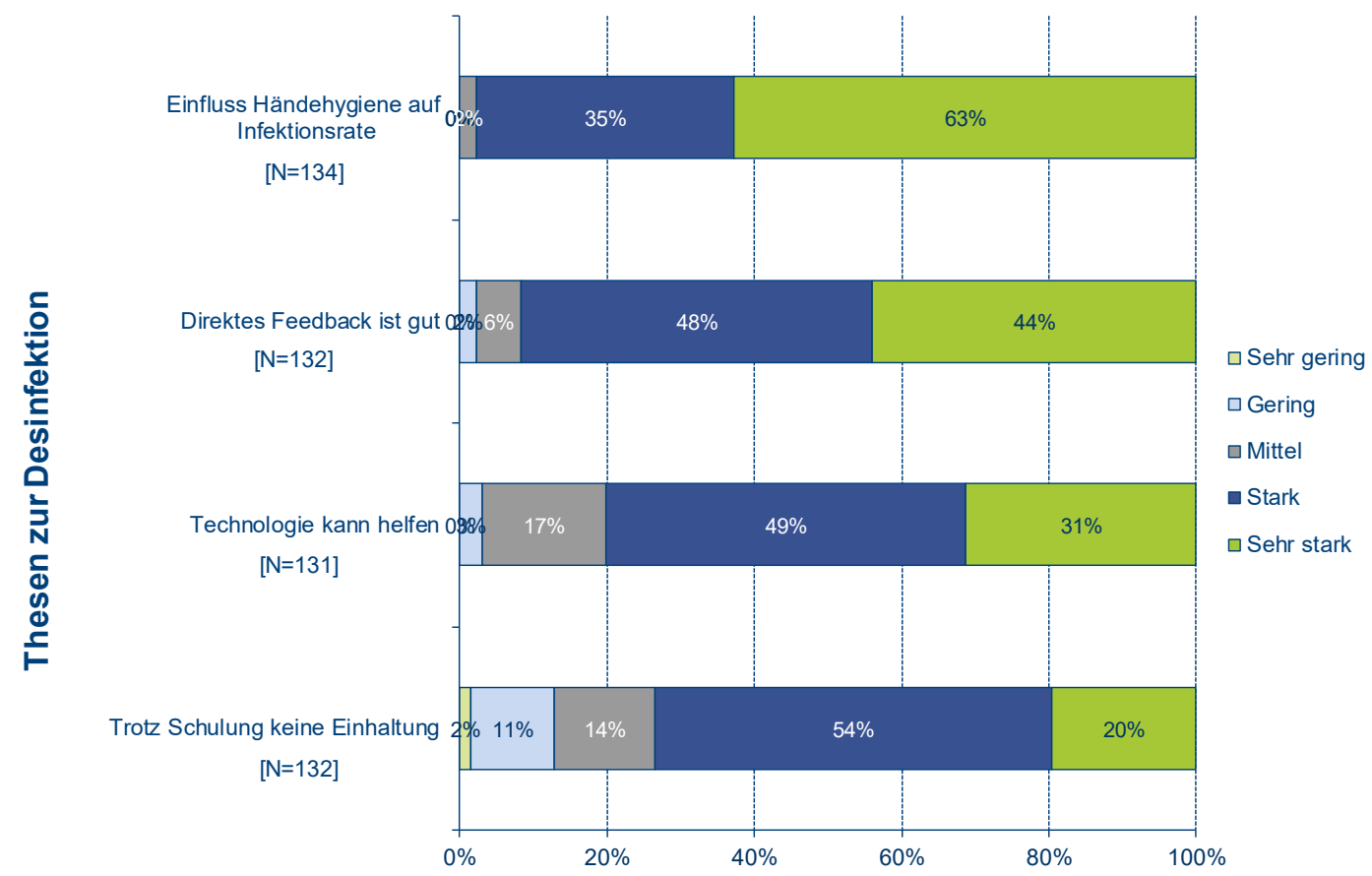

Abbildung 6: Thesen zur Desinfektion I

5. Es gibt zu wenige Kapazitäten in der Hygiene, um Beobachtungen/Observationen durchzuführen und die Compliance zu erhöhen.

$\rightarrow$ These wird teilweise bestätigt: 64 Prozent (22 Prozent sehr stark, 42 Prozent stark) haben nicht ausreichend Kapazitäten für Beobachtungen/Observationen zur Verfügung. Für 21 Prozent sind die verfügbaren Kapazitäten ausreichend.

6. Hygienefachkräfte werden teilweise nicht ernst genommen und können oft nicht auf Augenhöhe mit Betroffenen sprechen.

$\rightarrow$ These wird teilweise bestätigt: Allerdings sind es nur 53 Prozent (18 Prozent sehr stark, 34 Prozent stark), die Hygienefachkräfte als nicht ernst genommen wahrnehmen. 31 Prozent (7 Prozent sehr gering, 24 Prozent gering) widersprechen der These. 
7. Flächenhygiene oder die Einhaltung der Hygienepläne kann nicht oder nur sehr eingeschränkt überprüft werden, es sind keine Kapazitäten für diese Aufgabe vorhanden. Oft wird diese Verantwortung an die Hauswirtschaft delegiert.

$\rightarrow$ These wird teilweise bestätigt: 45 Prozent (11 Prozent sehr stark, 34 Prozent stark) halten die Einhaltung für kaum prüfbar und bestätigen, dass keine Kapazitäten hierfür vorhanden sind, sowie die Aufgabe oft an die Hauswirtschaft delegiert wird. 24 Prozent, enthalten sich der Aussage und 31 Prozent (8 Prozent sehr gering, 23 Prozent gering) verwerfen die These.

8. Für umfassendes Feedback liegen nicht ausreichend Daten vor.

$\rightarrow$ These wird teilweise bestätigt: 39 Prozent (11 Prozent sehr stark, 27 Prozent stark) bestätigen, dass nicht ausreichend Daten für umfassendes Feedback vorliegen. 31 Prozent (10 Prozent sehr gering, 21 Prozent gering) sind der Meinung, dass ausreichend Daten vorliegen.

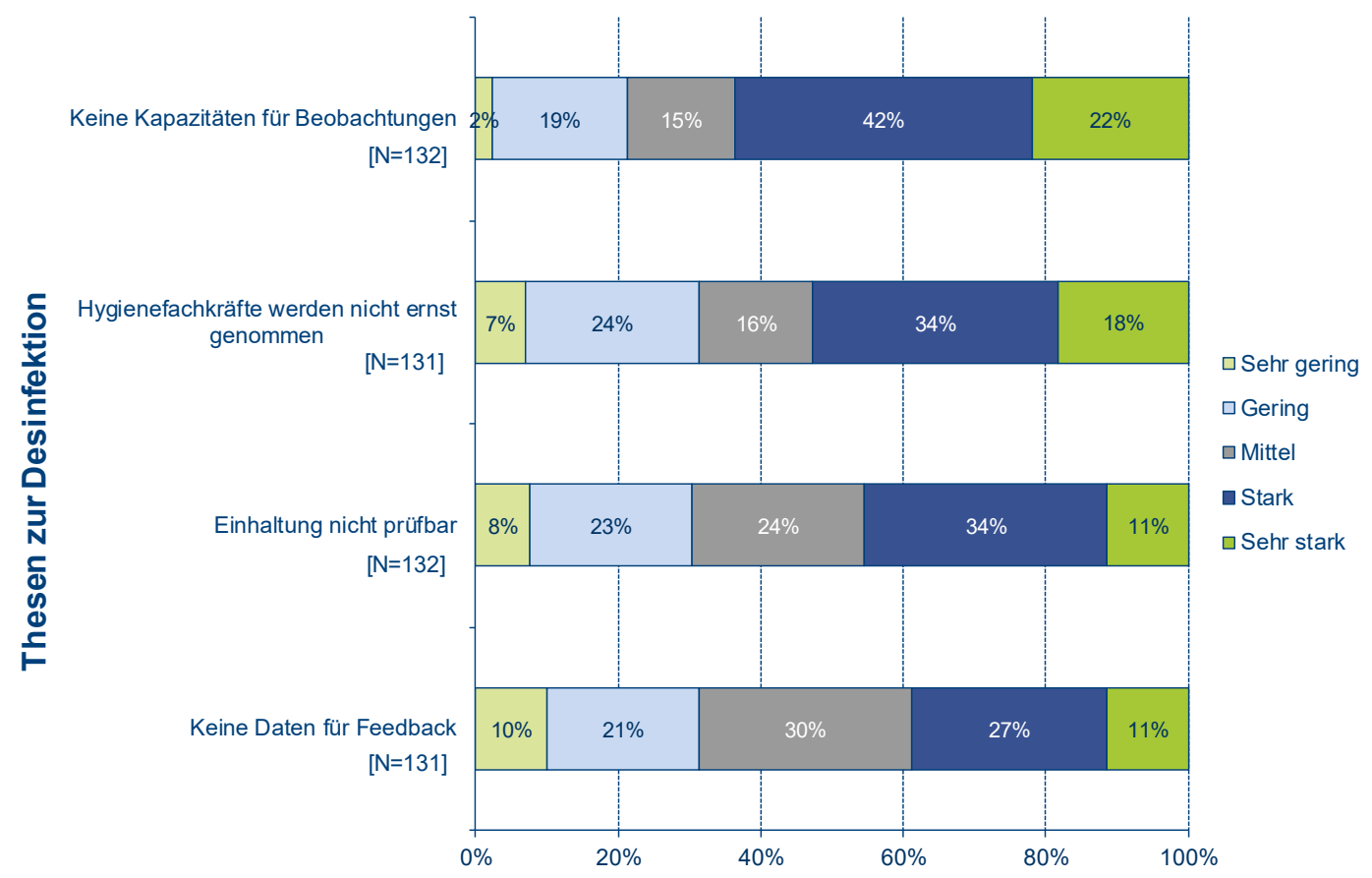

Abbildung 7: Thesen zur Desinfektion II

9. Das Kommunikationsniveau im Krankenhaus ist sehr wenig entwickelt.

$\rightarrow$ These wird teilweise bestätigt: 32 Prozent (15 Prozent sehr gering, 35 Prozent gering) halten das Kommunikationsniveau für wenig entwickelt. Die Hälfte der Probanden (15 Prozent sehr stark, 35 Prozent stark) widersprechen der These. 
10. Hygienefachkräfte und Hygieniker sind nicht geschult, um nachhaltige Verhaltensänderungen in Teams oder bei Individuen umsetzen zu können.

$\rightarrow$ These wird verworfen: 29 Prozent (10 Prozent sehr stark, 19 Prozent stark) sind der Meinung, Hygienefachkräfte und Hygieniker sind zu wenig geschult. 57 Prozent (17 Prozent sehr gering, 40 Prozent gering) der Probanden sind der Meinung, das Schulungsniveau ist ausreichend.

11. Händedesinfektion schädigt die Haut. Daher wird eine häufige Händedesinfektion vermieden.

$\rightarrow$ These wird verworfen: Nur 16 Prozent (5 Prozent sehr stark, 11 Prozent stark) der Probanden stimmen der These zu. 76 Prozent (47 Prozent sehr gering, 28 Prozent gering) empfinden häufiges Händedesinfizieren nicht als schädlich.

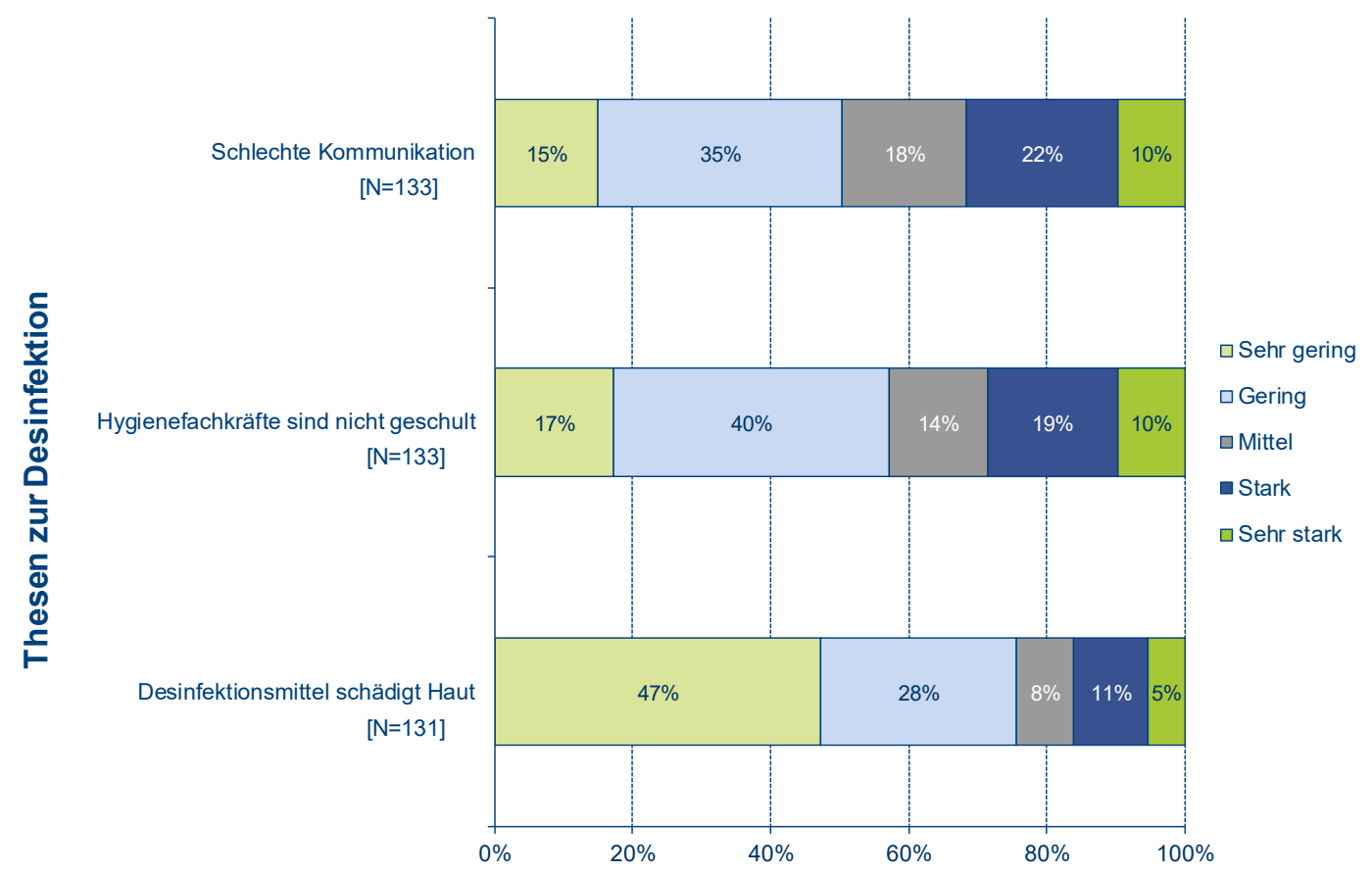

Abbildung 8: Thesen zur Desinfektion III 


\subsection{Kontrastierung}

Als Grundlage für die Kontrastierungen wurden die Einschätzungen der Probanden zu den Thesen zur Desinfektion im Krankenhaus herangezogen. Dabei stand die Korrelation der Antworten mit den Faktoren Form, Trägerschaft, Funktionsbereich, Bettenanzahl und Standort im Fokus. Die Ergebnisse resultieren aus der Betrachtung des Mittelwerts jeder These in Abhängigkeit des jeweiligen Parameters.

Während sich bei der Trägerschaft und Form des Krankenhauses kaum Unterschiede feststellen lassen, ist die Bettenanzahl und der Standort des Krankenhauses, sowie der Funktionsbereich des Probanden von Bedeutung.

\subsubsection{Bettenanzahl}

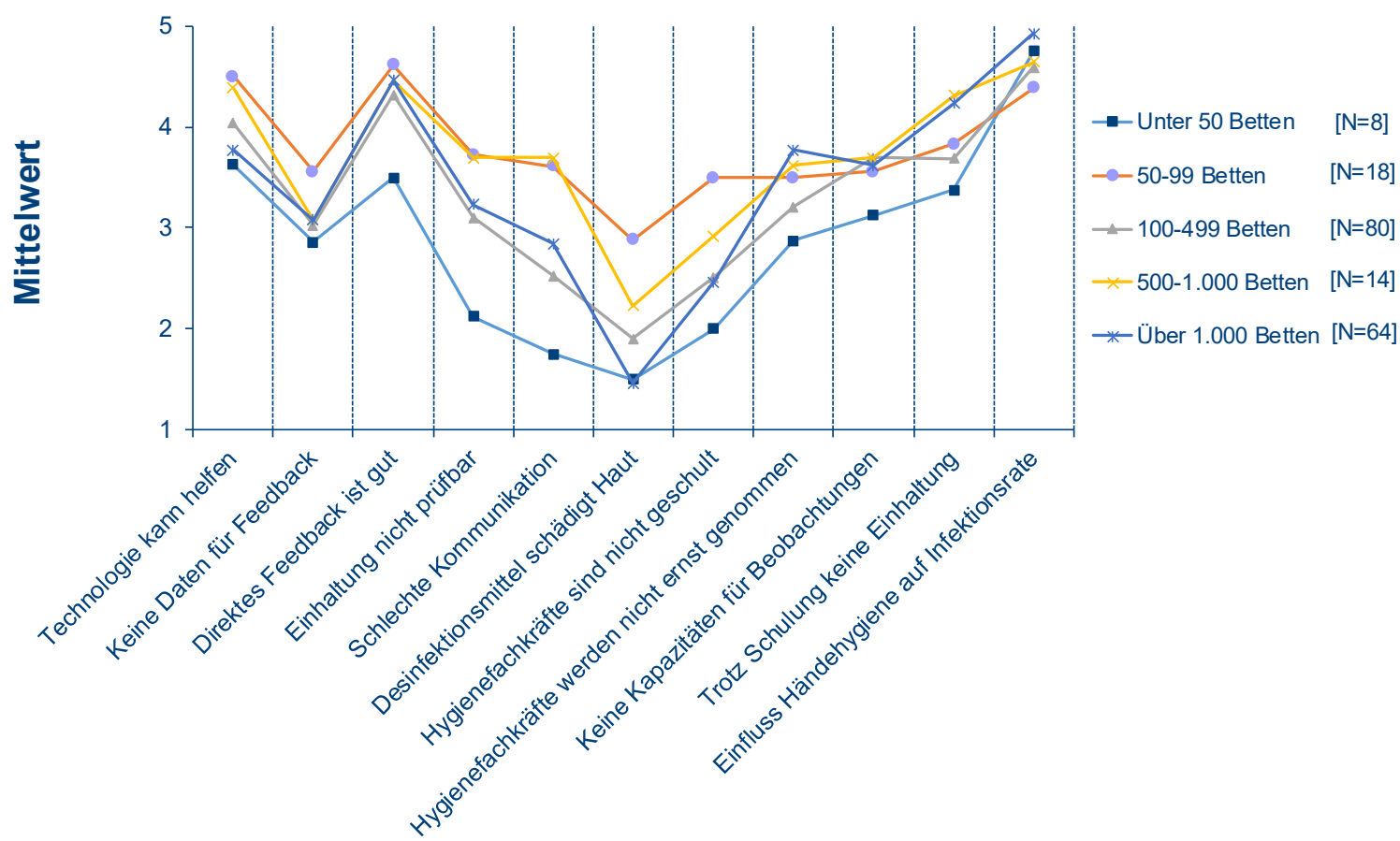

Thesen

\section{Abbildung 9: Kontrastierung Thesen und Anzahl Betten}

Abbildung 9 stellt den Mittelwert der Thesenbeurteilung im Kontrast zur Anzahl der Betten dar. Daraus lassen sich folgende Aussagen ableiten:

- Kleine Krankenhäuser, die weniger als 50 Betten zur Verfügung haben stimmen den Thesen im Allgemeinen weniger zu, als Krankenhäuser, die zwischen 50 und 99 Betten besitzen. 
- Das Kommunikationsniveau ist in kleinen Krankenhäusern weniger gut entwickelt, als in großen Krankenhäusern.

- In Krankenhäusern bis 50 Betten ist die Einhaltung leichter prüfbar, als in gröBeren Krankenhäusern.

- Die Meinungen zur Schädlichkeit des Desinfektionsmittels variieren stark.

\subsubsection{Standort}

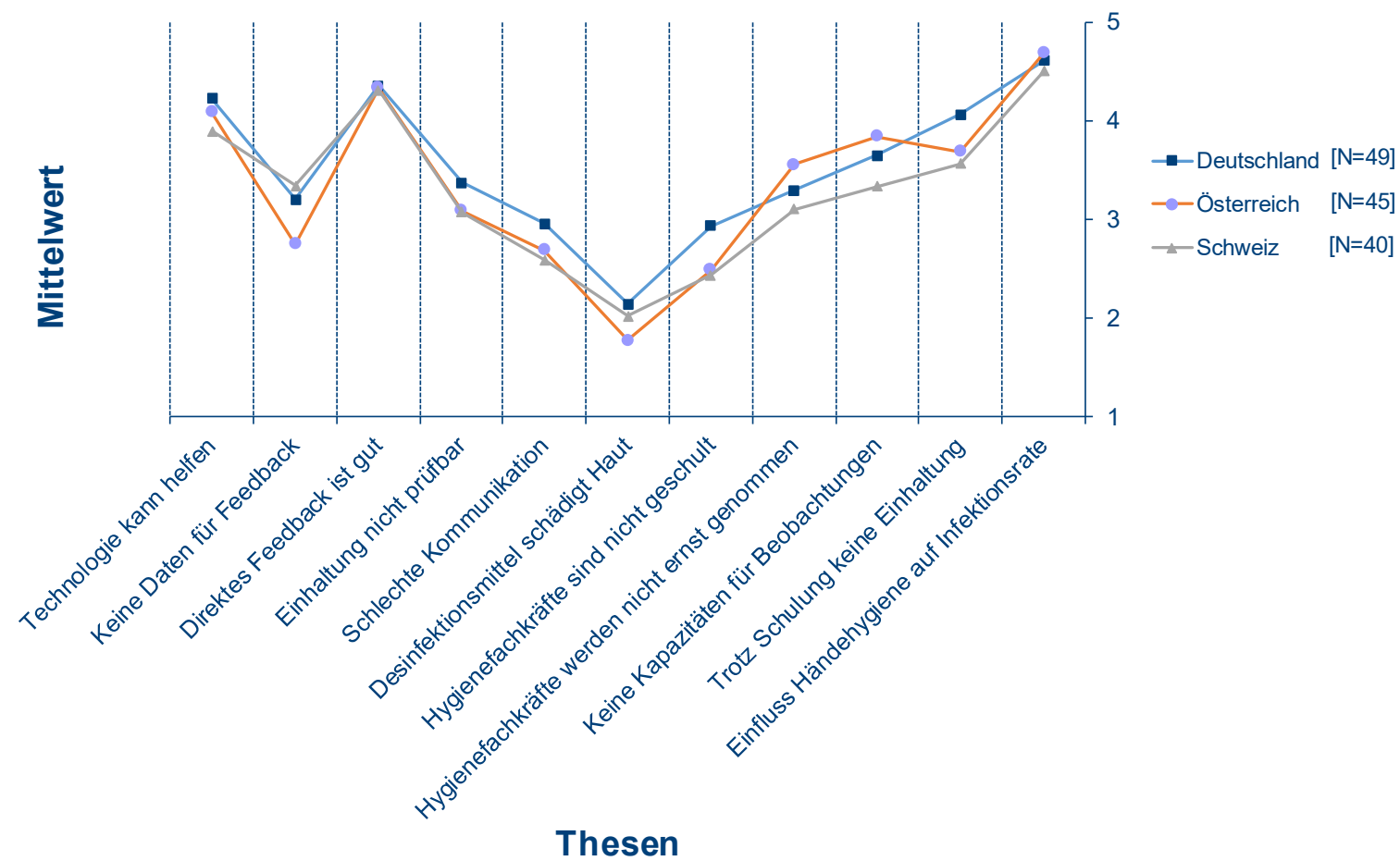

Abbildung 10: Kontrastierung Thesen und Standort 


\subsubsection{Funktionsbereich}

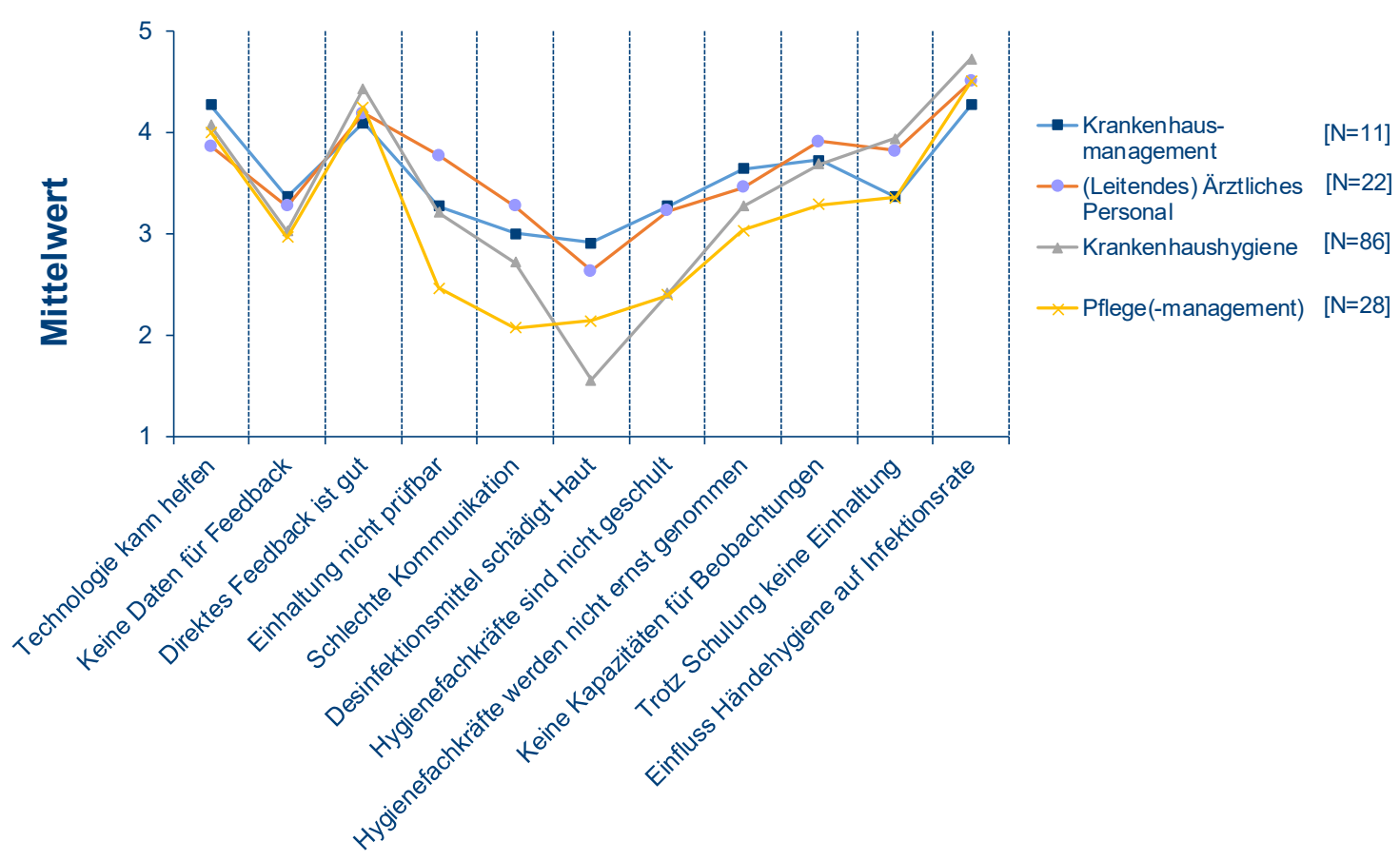

Thesen

\section{Abbildung 11: Kontrastierung Thesen und Funktionsbereich}

Zur Verdeutlichung und Übersichtlichkeit sind bei der Kontrastierung die Funktionen leitendes ärztliches Personal und ärztliches Personal in einer Kategorie abgebildet. Auch Pflege und Pflegemanagement wurden hier zusammengelegt.

Folgende Aussagen lassen sich aus Abbildung 6 treffen:

- Auch unter der Betrachtung des Funktionsbereiches sind die Meinungen zur Schädlichkeit von Desinfektionsmittel verschieden. Während die Krankenhaushygiene und die Pflege die These verwerfen, sind sich das Krankenhausmanagement und ärztliches Personal unschlüssig.

- Das ärztliche Personal empfindet die Regeleinhaltung als nicht prüfbar, wohingegen die Pflege tendenziell widerspricht.

- Außerdem ist das ärztliche Personal eher der Meinung, dass das Kommunikationsniveau zu wenig entwickelt ist, als die Pflege. 


\section{Fragen zur Desinfektion}

Die folgenden Unterkapitel befassen sich mit allgemeinen Fragen zur Desinfektion, wie bspw. Schulungen, Gründe für Nicht-Einhaltung, sowie Einschätzungen zur Compliance im Krankenhaus.

\subsection{Einhaltungsgrad der Regeln zur Händedesinfektion}

63 Prozent der Befragten schätzen den Einhaltungsgrad der Regeln zur Händedesinfektion zwischen 51 und 75 Prozent. 21 Prozent schätzen den Einhaltungsgrad auf unter 50 Prozent und 16 Prozent schätzen inn auf über 75 Prozent.

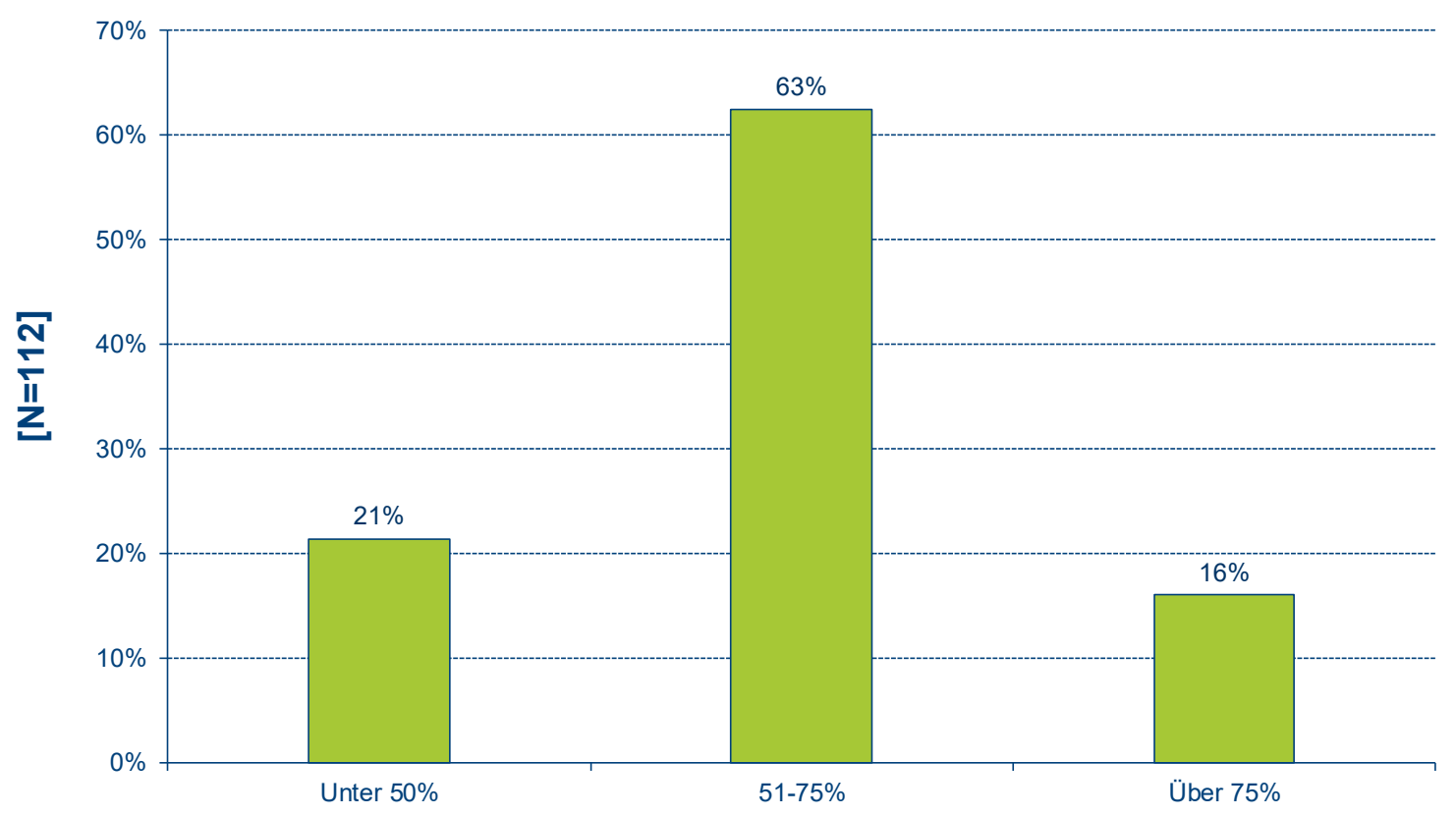

Einhaltungsgrad der Regeln zur Händedesinfektion 


\subsection{Rate nosokomialer Infektionen im Krankenhaus}

Die Befragung zeigt, dass etwas über die Hälfte (53 Prozent) der befragten Probanden die Rate nosokomialer Infektionen in Ihrem Krankenhaus pro Jahr auf 1 bis 5 Prozent schätzen. 15 Prozent schätzen diese auf unter ein Prozent und jeweils 16 Prozent schätzen auf eine Rate zwischen 5 und 10 bzw. über 10 Prozent.

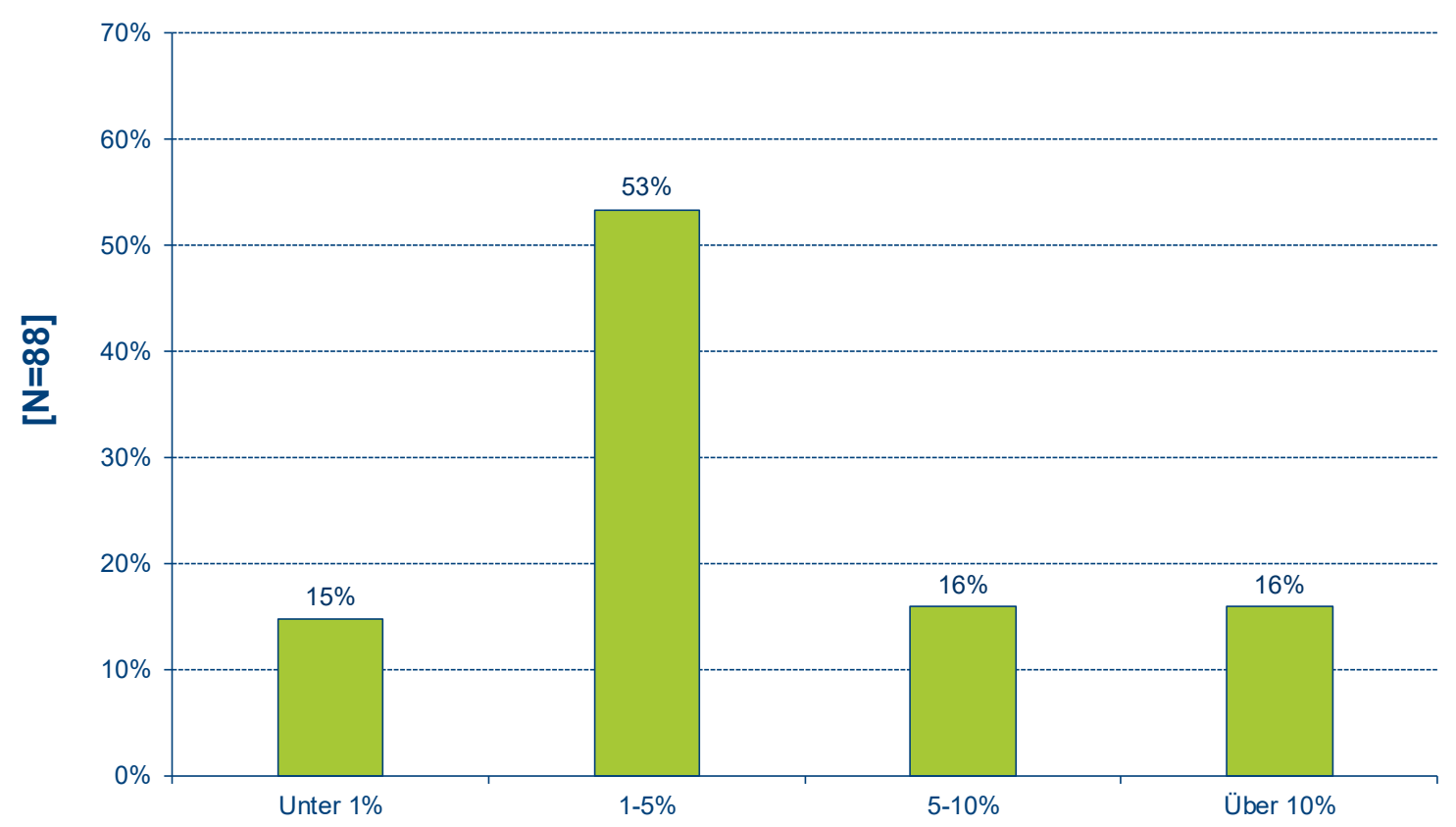

Nosokomiale Infektionsrate pro Jahr

\section{Abbildung 13: Nosokomiale Infektionsrate pro Jahr}

\subsection{Schulungen pro Jahr}

Die Antworten auf die Anzahl der Schulungen pro Jahr schwanken deutlich. Hierbei ist leider nicht ersichtlich, inwiefern sich die Angaben auf Mitarbeiter, Station oder das gesamte Krankenhaus beziehen. Eine klare Aussage lässt sich folglich nicht daraus schließen. 


\subsection{Schulungsthemen}

94 Prozent bestätigen die Frage, ob das Thema Händedesinfektion geschult wird, mit der Antwort: Ja. 79 Prozent der befragten Probanden versichern außerdem, dass Flächenhygiene und 68 Prozent, dass Compliance allgemein geschult wird. 56 Prozent geben zusätzlich sonstige Themen an.

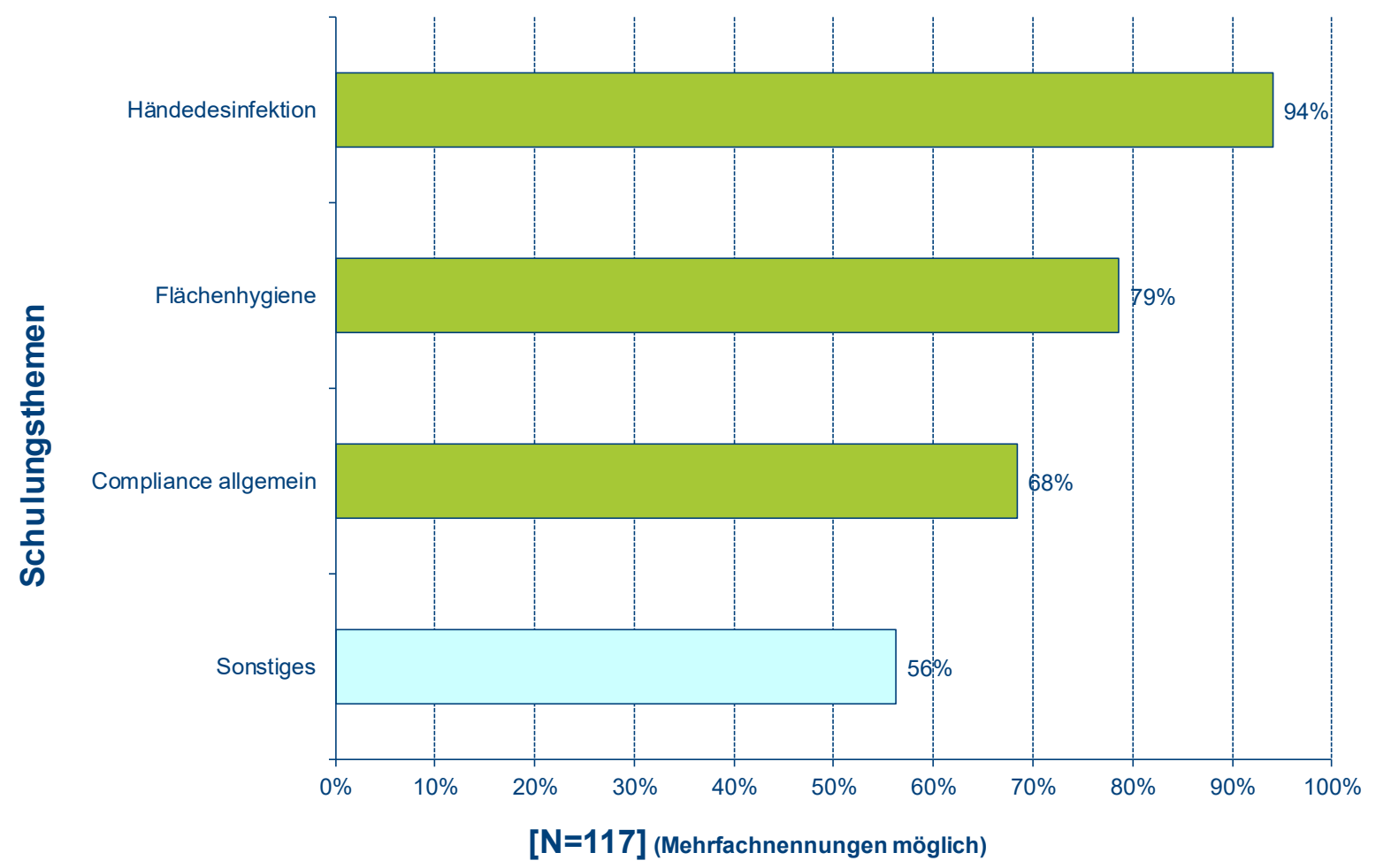

Abbildung 14: Schulungsthemen 


\subsection{Art der Schulungen}

Nun wird geprüft, um welche Art der Schulung es sich handelt. Hier sind Mehrfachnennungen möglich. 81 Prozent werden intern durch eigene Mitarbeiter bei Präsenzschulungen geschult. Weitere 37 Prozent werden ebenfalls durch Präsenzschulungen über die Themen informiert, jedoch über externe Coaches o.Ä. 9 Prozent der Krankenhäuser geben an, es werden Präsenzschulungen außer Haus in beispielsweise Tagungshotels gehalten, 26 Prozent der Schulungen sind Online-Schulungen/Webinare. 5 Prozent werden auf sonstige Arten geschult.

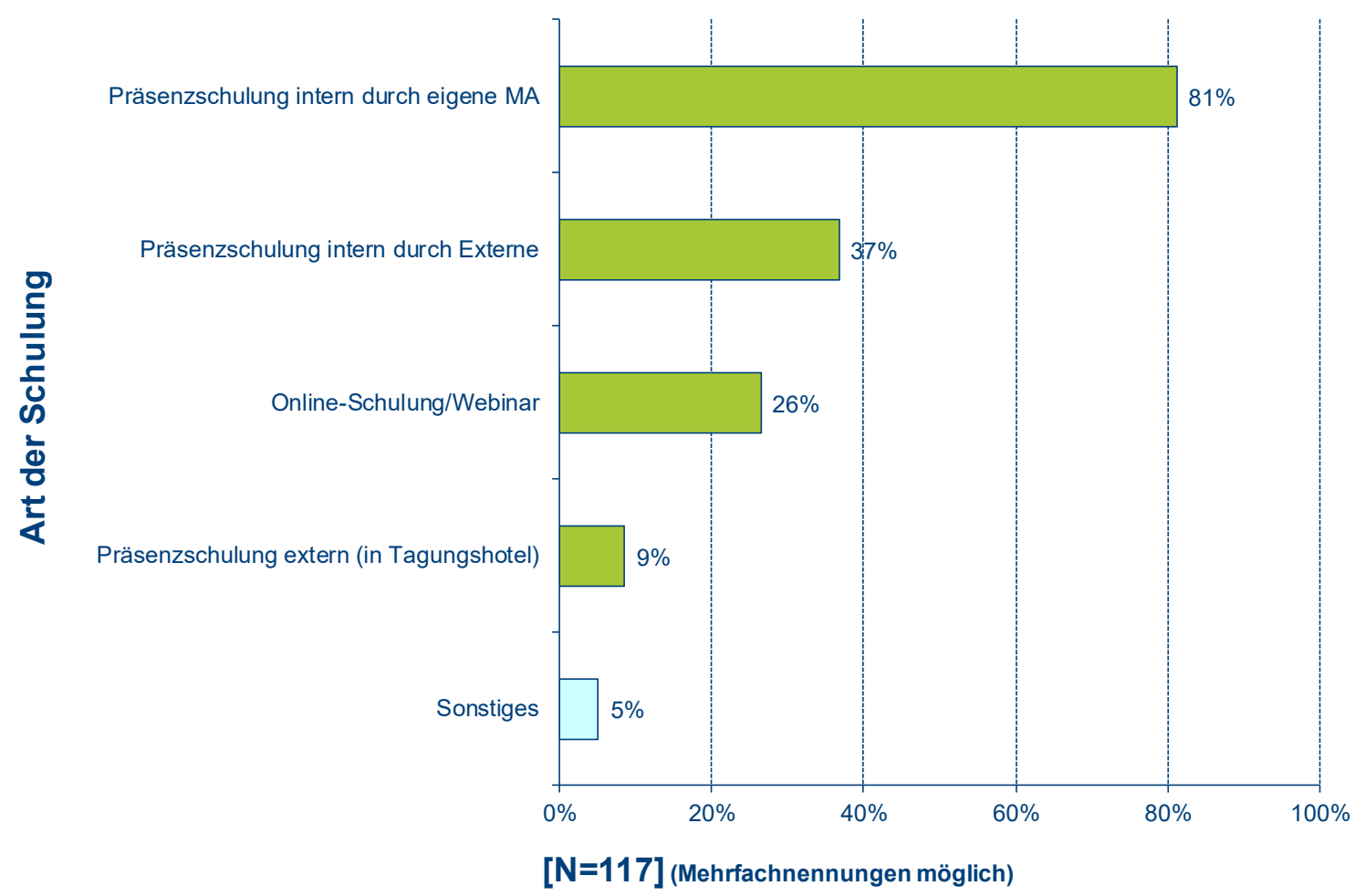

Abbildung 15: Art der Schulungen 


\subsection{Verbesserungsbedarf bezüglich Schulungen}

Bei der Frage, in welchen Themen Verbesserungsbedarf bezüglich der Schulungen besteht, geben 68 Prozent Compliance im Algemeinen an. Dass das Thema Flächendesinfektion Verbesserungsbedarf hat, stimmen 41 Prozent zu. 40 Prozent halten das Thema Händedesinfektion für verbesserungswürdig.

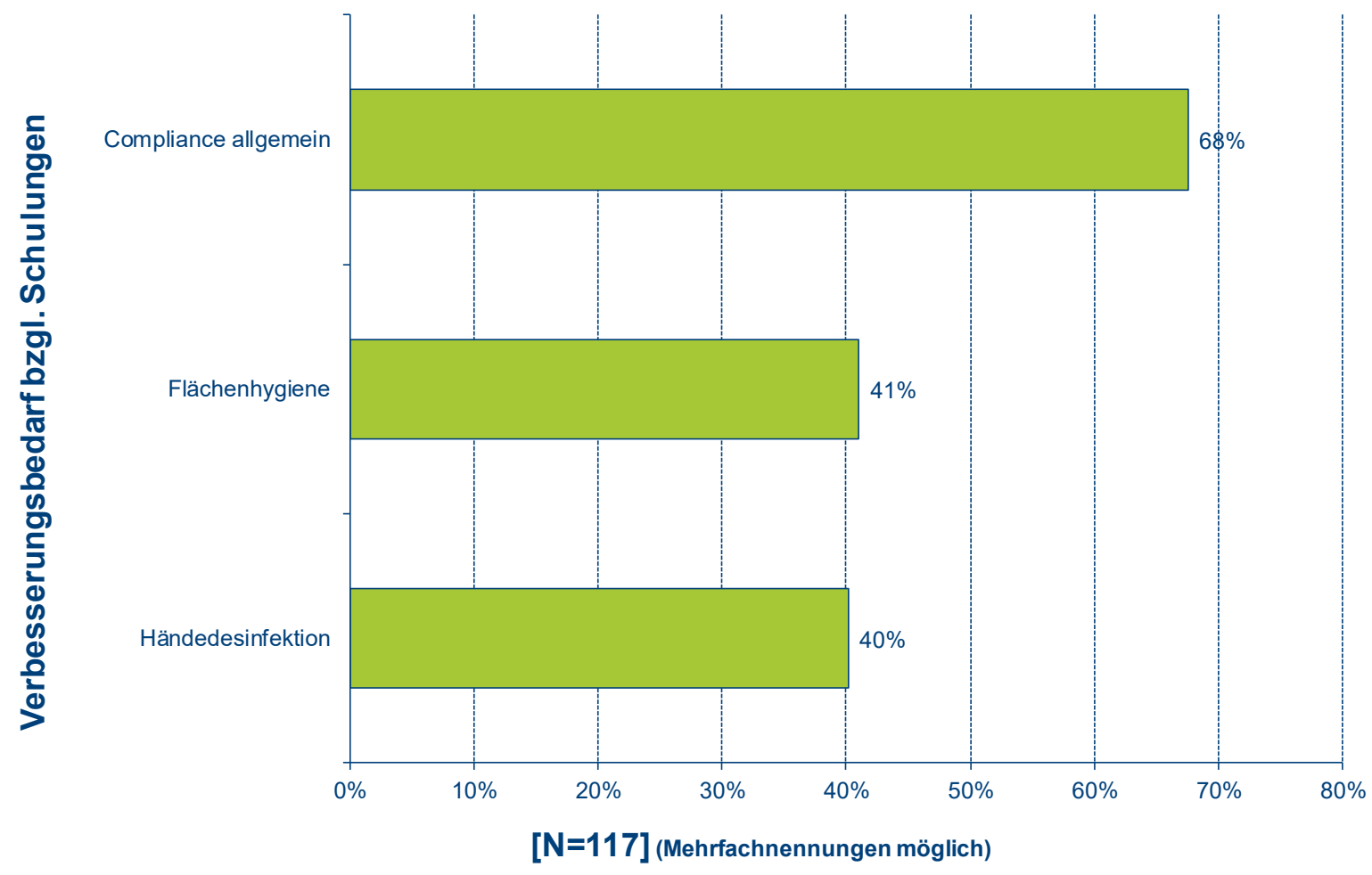

Abbildung 16: Verbesserungsbedarf bezüglich Schulungen 


\subsection{Interesse an begleitenden Schulungen durch die Industrie}

Die Probanden haben die Möglichkeit, ihr Interesse an begleiteten Schulungen durch die Industrie zu zeigen. Hier stehen Präsenzschulungen und Online-Schulungen/Webinare zur Auswahl. Interesse an Präsenzschulungen durch die Industrie haben 37 Prozent und 63 Prozent halten dies für überflüssig. Wohingegen an Online-Schulungen/Webinaren 41 Prozent interessiert wären und etwas mehr als die Hälfte (59 Prozent) kein Interesse daran haben.

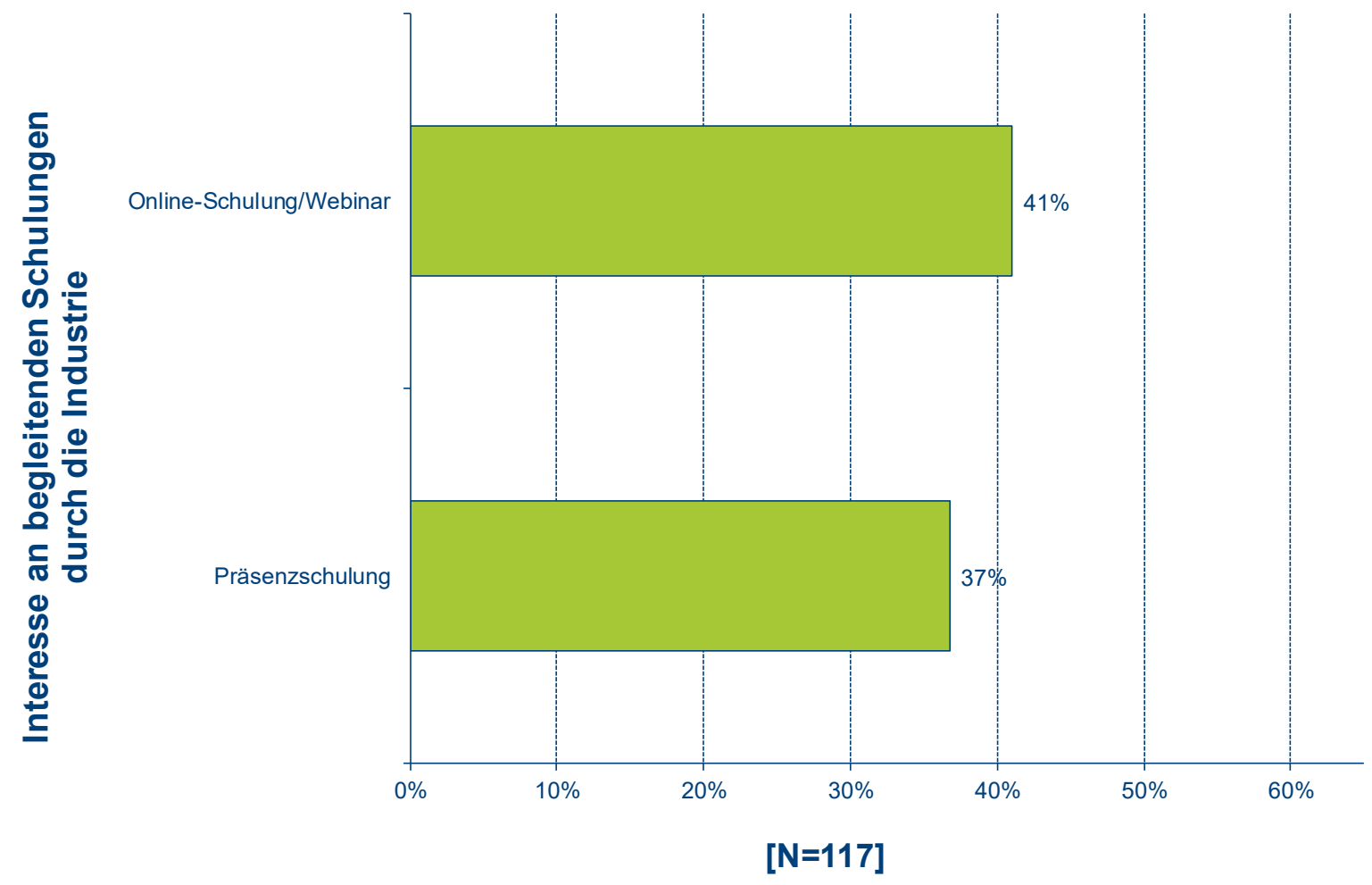

Abbildung 17: Interesse der Krankenhäuser an begleitenden Schulungen durch die Industrie 


\subsection{Einschätzung der Kapazitäten für Observationen/Beobachtun- gen der Regeleinhaltung}

Um herauszufinden wie die Kapazitäten für Observationen/Beobachtungen der Regeleinhaltung eingeschätzt werden, wurden weiter sämtliche Teilnehmer befragt. Die Umfrage zeigt, dass die Hälfte (50 Prozent) die Kapazitäten mittelmäßig einschätzen, 15 Prozent schlecht und 10 Prozent sehr schlecht. Auf der anderen Seite schätzen 18 Prozent die Kapazitäten als gut ein und weitere 6 Prozent als sehr gut.

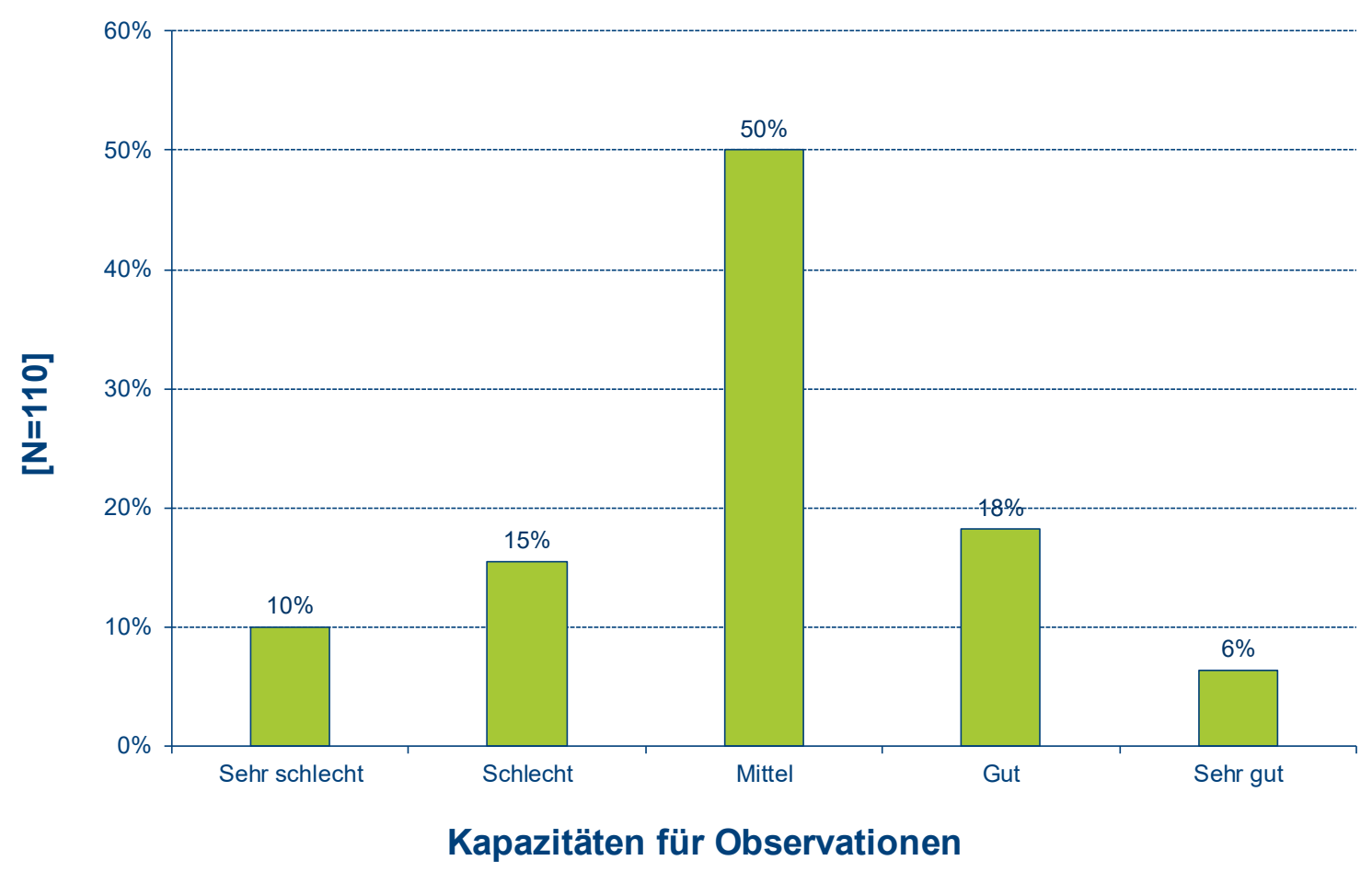

Abbildung 18: Einschätzung der Kapazitäten für Observationen 


\subsection{Akzeptanz von Pflegekräften im Vergleich zu ärztlichem Per- sonal}

Die Probanden werden nun befragt, wie sie die Akzeptanz von Pflegekräften im Vergleich zu ärztlichem Personal einschätzen. Die Umfrage zeigt, dass genau ein Drittel die Akzeptanz von Pflegekräften als höher einschätzen und 26 Prozent als viel höher. Dem gegenüber sind sich 25 Prozent einig, dass die Akzeptanz von Pflegekräften im Vergleich zu ärztlichem Personal mittel ist, 10 Prozent, dass sie gering ist und 2 Prozent, dass die Akzeptanz viel geringer ist.

Es besteht allerdings der Verdacht, dass die Frage so interpretiert wurde, dass es sich nicht auf die Akzeptanz der Person an sich bezieht, sondern auf die Akzeptanz der Regelungen.

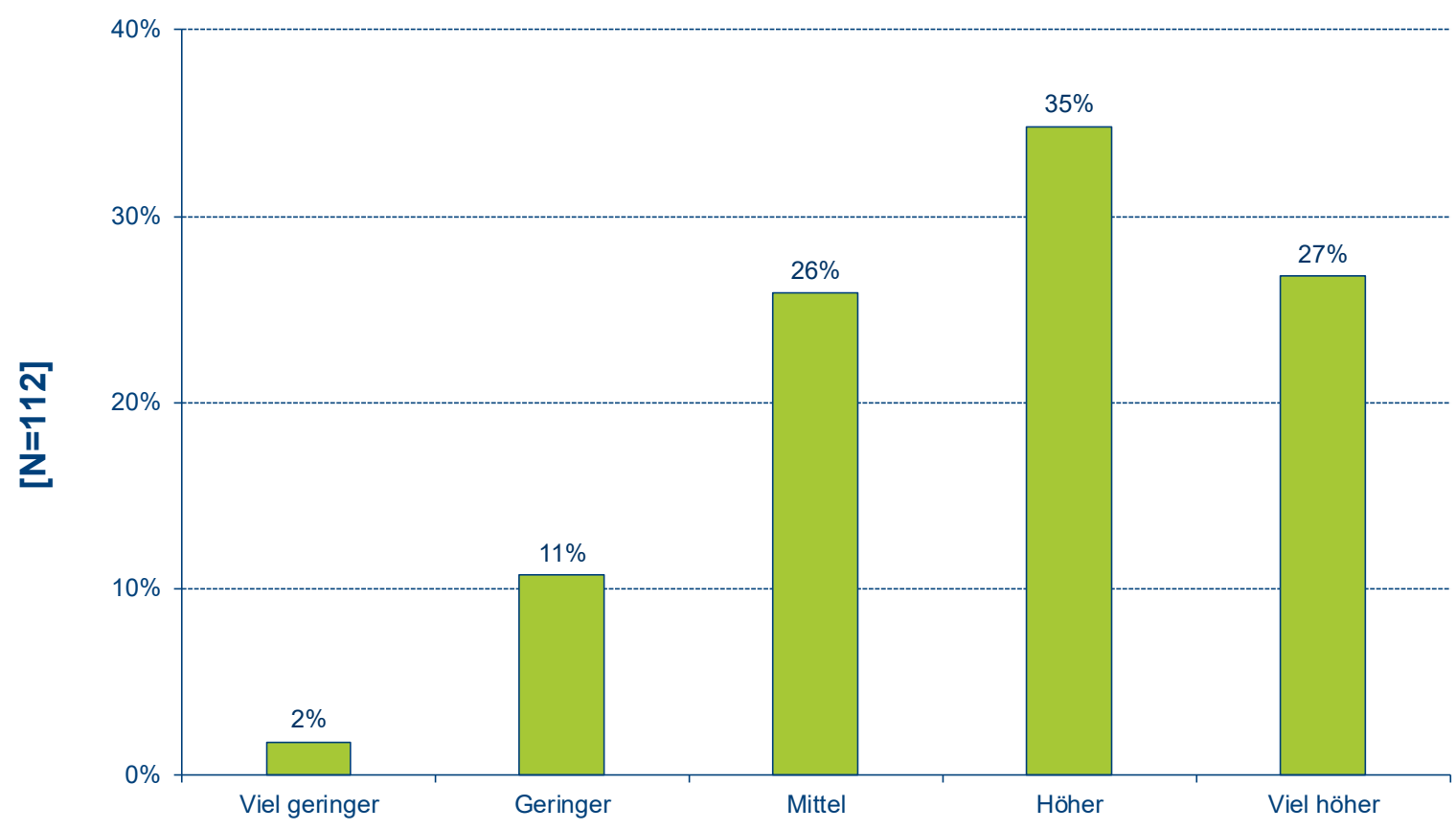

Akzeptanz von Pflegekräften im Vergleich zu ärztlichem Personal 


\subsection{Einschätzung Hygienefachkräfte/-teams}

Nun werden die Probanden befragt, wie Sie die Hygienefachkräfte/-team bezüglich ausgewählten Kriterien einschätzen. Insgesamt 77 Prozent (45 Prozent sehr hoch, 32 Prozent hoch) schätzen das Schulungsniveau als hoch bzw. sehr hoch ein. Nur 10 Prozent (0 Prozent sehr gering, 10 Prozent gering) sind der Meinung, das Schulungsniveau sei zu wenig entwickelt. Ähnlich fallen die Ergebnisse bezüglich der Änderungsbereitschaft von Hygienefachkräften aus. 70 Prozent (26 Prozent sehr hoch, 44 Prozent hoch) halten die Änderungsbereitschaft für hoch, wohingegen 12 Prozent (1 Prozent sehr gering, 10 Prozent gering) anderer Meinung sind. Das Kriterium Organisation bei Hygienefachkräften/-teams schätzen 64 Prozent (25 Prozent sehr gut, 38 Prozent gut) als gut bzw. sehr gut ein. 12 Prozent (6 Prozent sehr schlecht, 6 Prozent schlecht) halten die Organisation für (sehr) schlecht. 32 Prozent (4 Prozent sehr gut, 28 Prozent gut) sind der Meinung, dass die personelle Ausstattung der Hygienefachteams gut sei. Für 29 Prozent (10 Prozent sehr schlecht, 19 Prozent schlecht) ist dies nicht der Fall. Das Zeitbudget wird nur mit 16 Prozent (2 Prozent sehr hoch, 14 Prozent sehr hoch) als ausreichend eingeschätzt. 45 Prozent (13 Prozent sehr gering, 33 Prozent gering) sind der Meinung, dass das Zeitbudget gering ist.

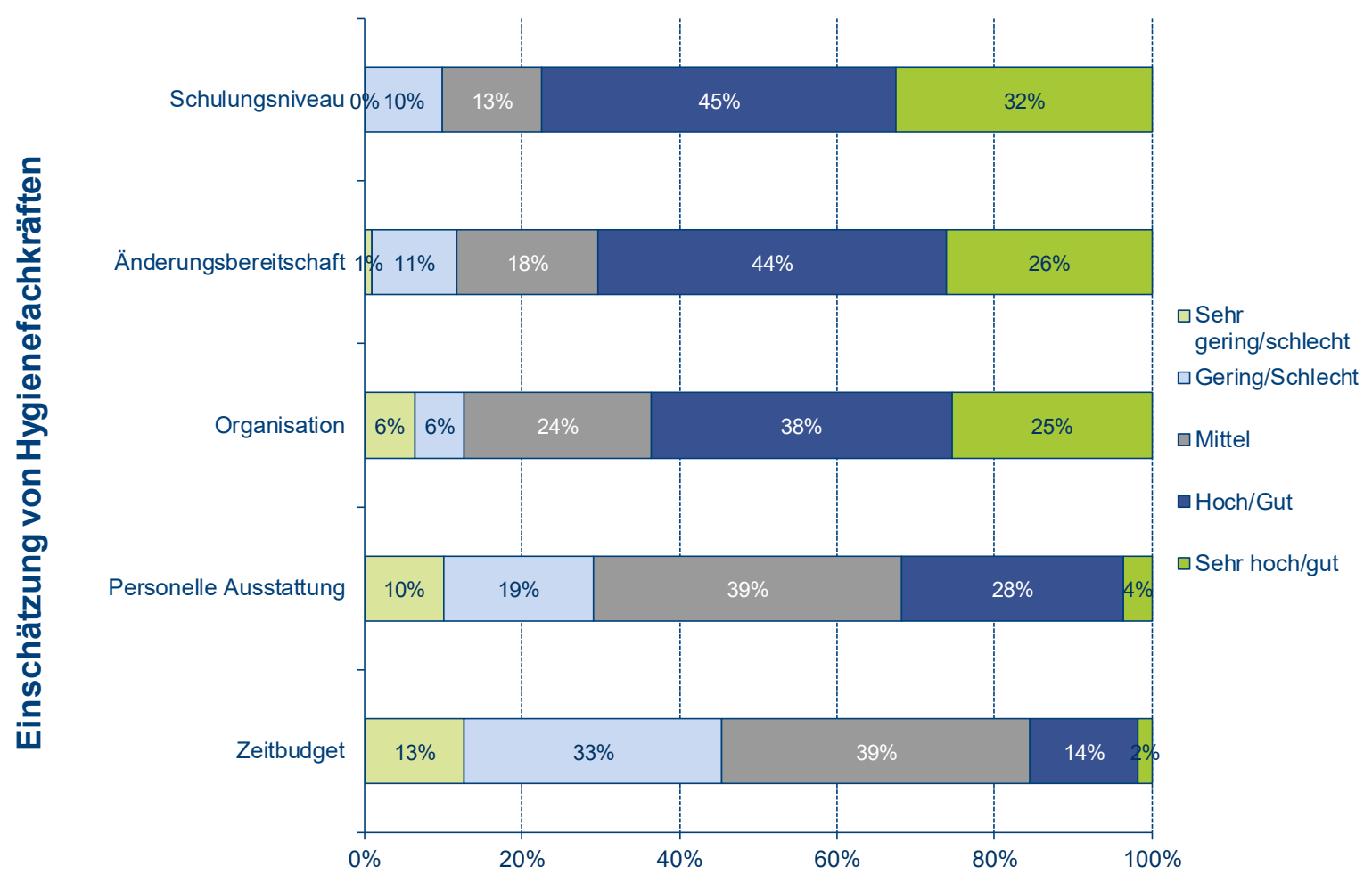




\subsection{Gründe für die Nicht-Einhaltung der WHO-Regeln zur Hände- desinfektion}

Hierbei wurden den Probanden 11 mögliche Gründe vorgeschlagen und gebeten, diese mit „ja“ oder "nein“ zu beantworten. Dabei sehen 80 Prozent der Probanden den Faktor Stress, und 59 Prozent die fehlende Zeit als Hauptursachen. 50 Prozent begründen die Unkenntnis als Grund für die Nichteinhaltung, 47 Prozent damit, dass es andere auch nicht machen und 43 Prozent, dass zu wenig Personal vorhanden ist. Ferner geben 40 Prozent an, dass es schädlich für die Hände sei. 23 Prozent sehen die Ursache in einem zu geringen Ausbildungs- und Schulungsniveau und 22 Prozent in der weitläufigen Verteilung der Spender. 14 Prozent sind davon überzeugt, dass die Regeln nicht sinnvoll sind. Zuletzt sind es 8 Prozent, die der Meinung sind, das Desinfektionsmittel sei häufig leer und 6 Prozent empfinden die Vorschriften als Anweisung von oben.

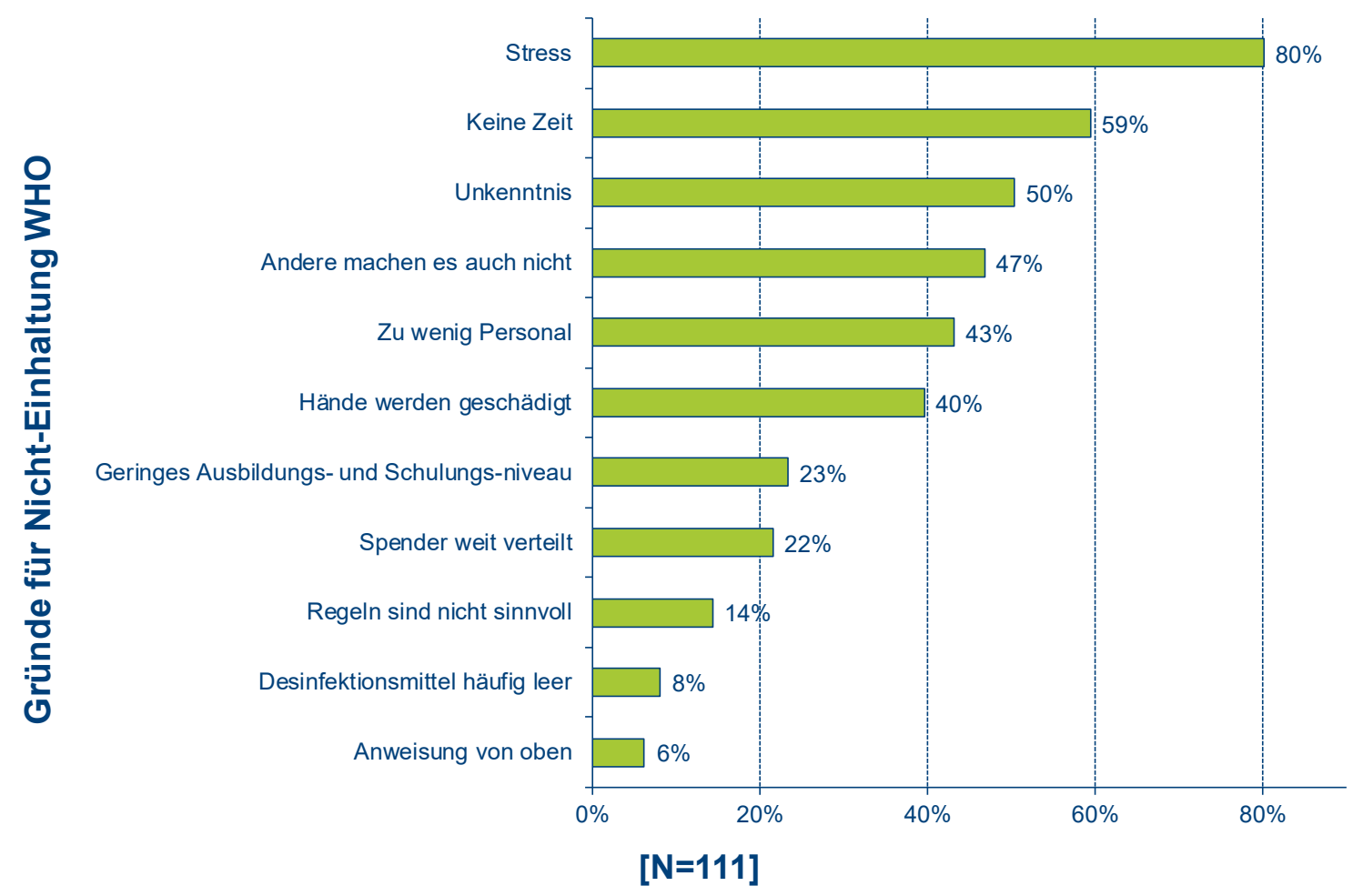

Abbildung 21: Gründe für die Nicht-Einhaltung der WHO-Regeln 


\subsection{Einschätzung des Kommunikationsniveaus im Krankenhaus}

Bei der Frage, wie die Probanden das Kommunikationsniveau im Krankenhaus einschätzen, hatten sie fünf Möglichkeiten von sehr schlecht bis sehr gut zu bewerten. Dabei gab 1 Prozent an, dass das Kommunikationsniveau sehr schlecht ist, 13 Prozent, dass es schlecht ist und 37 Prozent, dass es ausreichend ist. 39 Prozent schätzen das Kommunikationsniveau als gut ein und 5 Prozent als sehr gut. Die übrigen 5 Prozent haben zu dieser Frage keine Bewertung abgegeben.

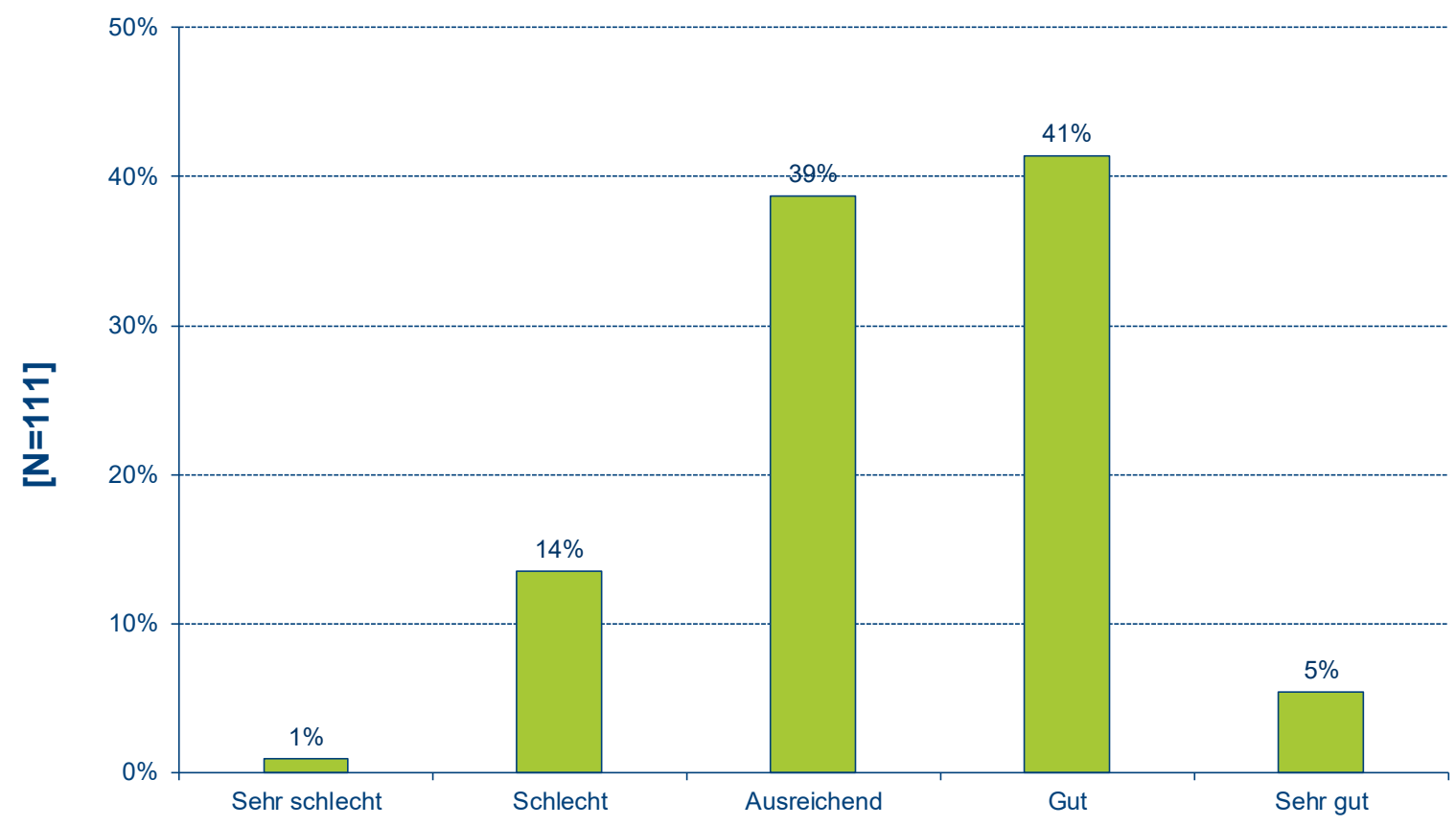

Kommunikationsniveau 


\subsection{Compliance-Quote im Bereich Flächendesinfektion}

Die Compliance-Quote im Bereich Flächendesinfektion wurde von 20 Prozent der Befragten mit unter 50 Prozent bewertet, von 47 Prozent mit 51-75 Prozent bewertet und von 33 Prozent mit über 75 Prozent bewertet. Daraus lässt sich ein Median von 70, eine Standardabweichung von 18, ein Minimum von 10 und ein Maximum von 95 berechnen.

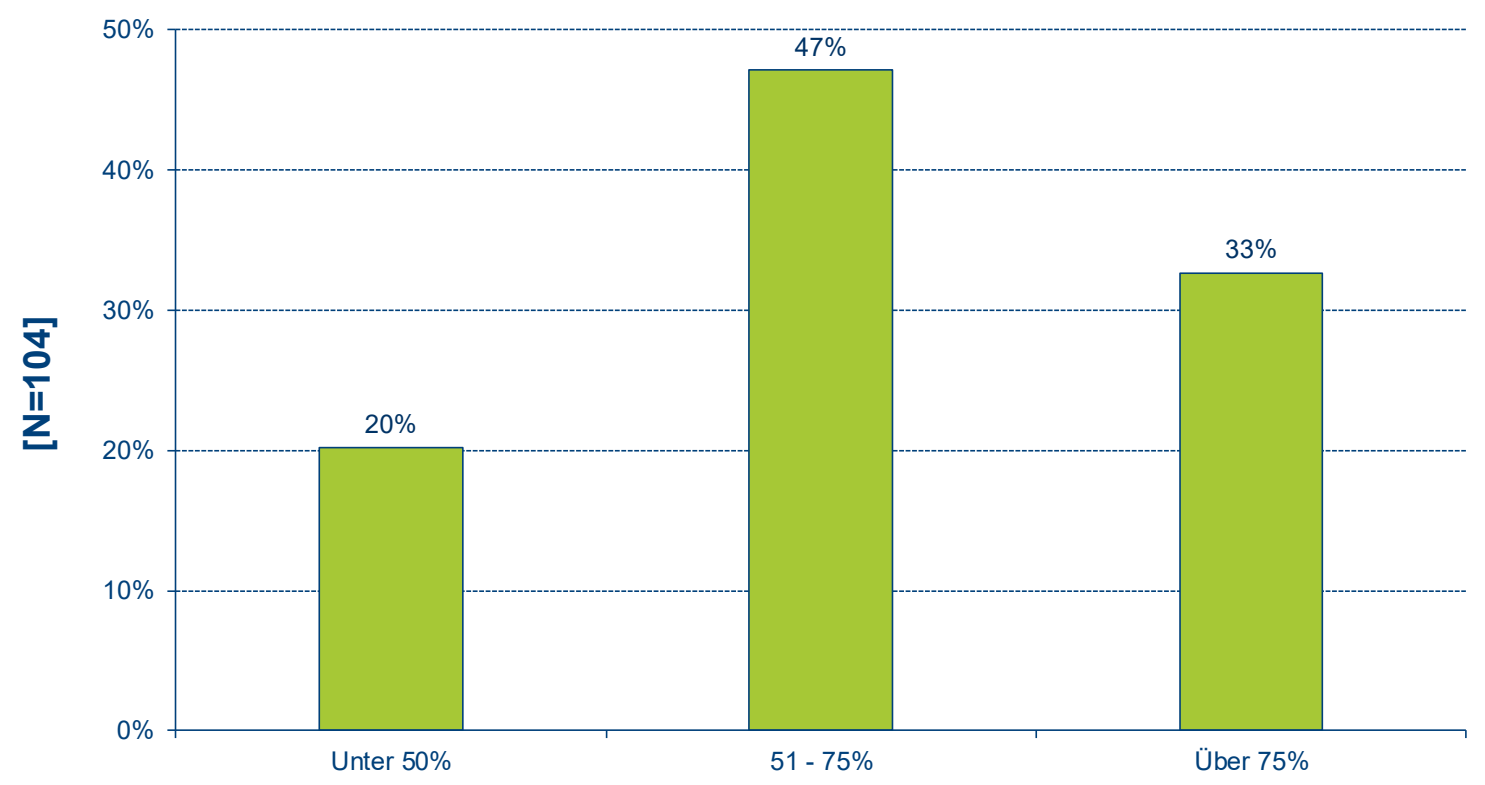

Compliance-Quote im Bereich Flächendesinfektion 


\subsection{Drei Aufgabenträger, die Flächendesinfektion durchführen}

Dieser Umfrage lässt sich entnehmen, dass nur 1 Prozent der Führungskräfte, sowie 1 Prozent des Medizinisch-technischen Personals und 1 Prozent der Hygienebeauftragten Flächendesinfektion durchführen. Das Facility Management führt bei 3 Prozent die Flächendesinfektion durch, das Stationspersonal inklusive Abteilungshelfer bei 5 Prozent und die (Assistenz/Fach-)Ärzte beziehungsweise Therapeuten bei 7 Prozent der Befragten. Mit großer Mehrheit lässt sich der Umfrage entnehmen, dass das Pflegepersonal mit 37 Prozent und die Hauswirtschaft bzw. Reinigung mit 44 Prozent für die Flächendesinfektion zuständig sind.

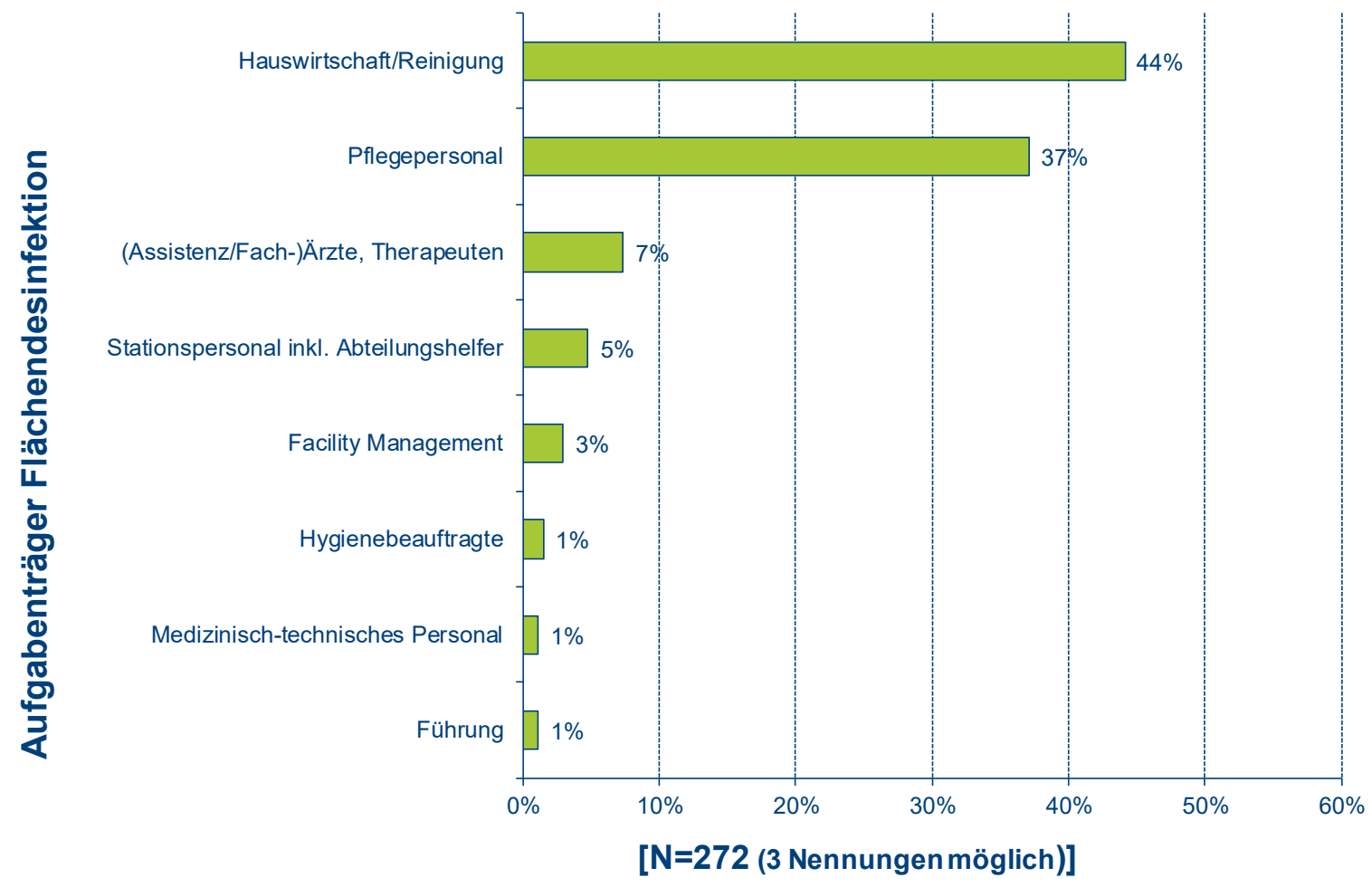

Abbildung 24: Aufgabenträger der Flächendesinfektion 


\subsection{Bewertung von Aussagen zu Feedback zum Krankenhaus}

Diese Frage diente dazu, die Wirkung von Feedbacks zu bewerten. Den Probanden wurden dabei fünf Aussagen gegeben, dies von sehr schlecht beziehungsweise gering, bis sehr hoch beziehungsweise gut oder viel zu bewerten. Ein direktes Feedback schätzen 61 Prozent als sehr hoch beziehungsweise gut ein, ein direktes Feedback geben wird von 44 Prozent als mittelmäßig und von 34 Prozent als hoch eingeschätzt und dass nur ausgewählten Personen ein direktes Feedback gegeben wird, bewerteten 33 Prozent mit gut. Einen Raum für Analyse und Reflektion bewerteten 27 Prozent mit gut und sogar fast die Hälfte der Befragten, 45 Prozent mit mittel.

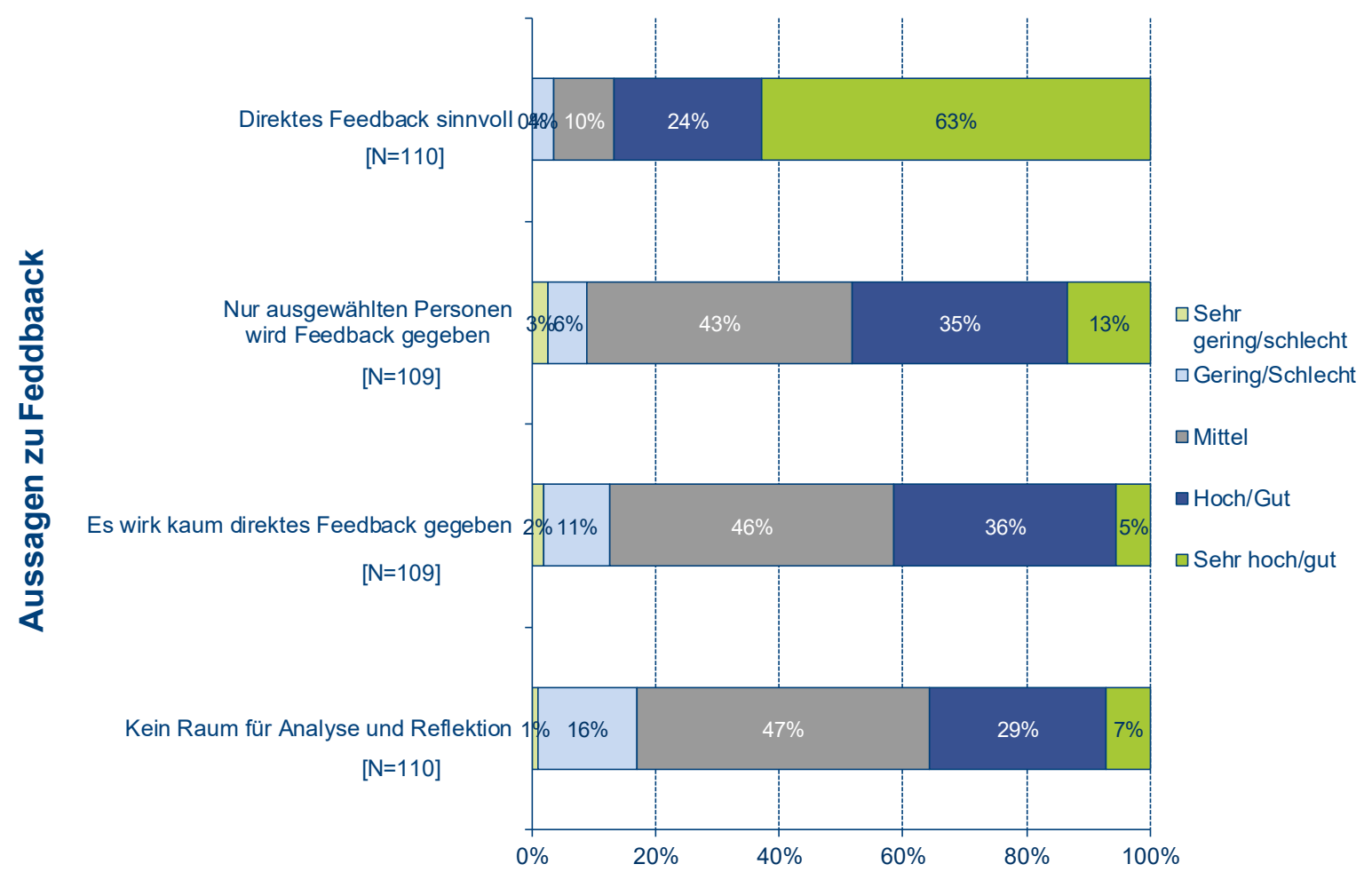

Abbildung 25: Aussagen zu Feedback im Krankenhaus 


\subsection{Drei Feedbackarten}

Im unteren Bereich mit 1-5 Prozent werden die Feedbackarten Qualitätszirkel, Führungskräftesitzungen, Betriebliches Vorschlagswesen, Audits und Hygienevisiten, Jahresgespräch beziehungsweise Jour Fix und Feedbacks aus Schulungen bewertet. Die Publikation von Testergebnissen wurde mit 11 Prozent bewertet, Teamsitzungen mit 13 Prozent und Umfragen beziehungsweise Interviews mit 16 Prozent. Mitarbeitergespräche wurden mit dem höchsten Prozentsatz von 39 Prozent bewertet.

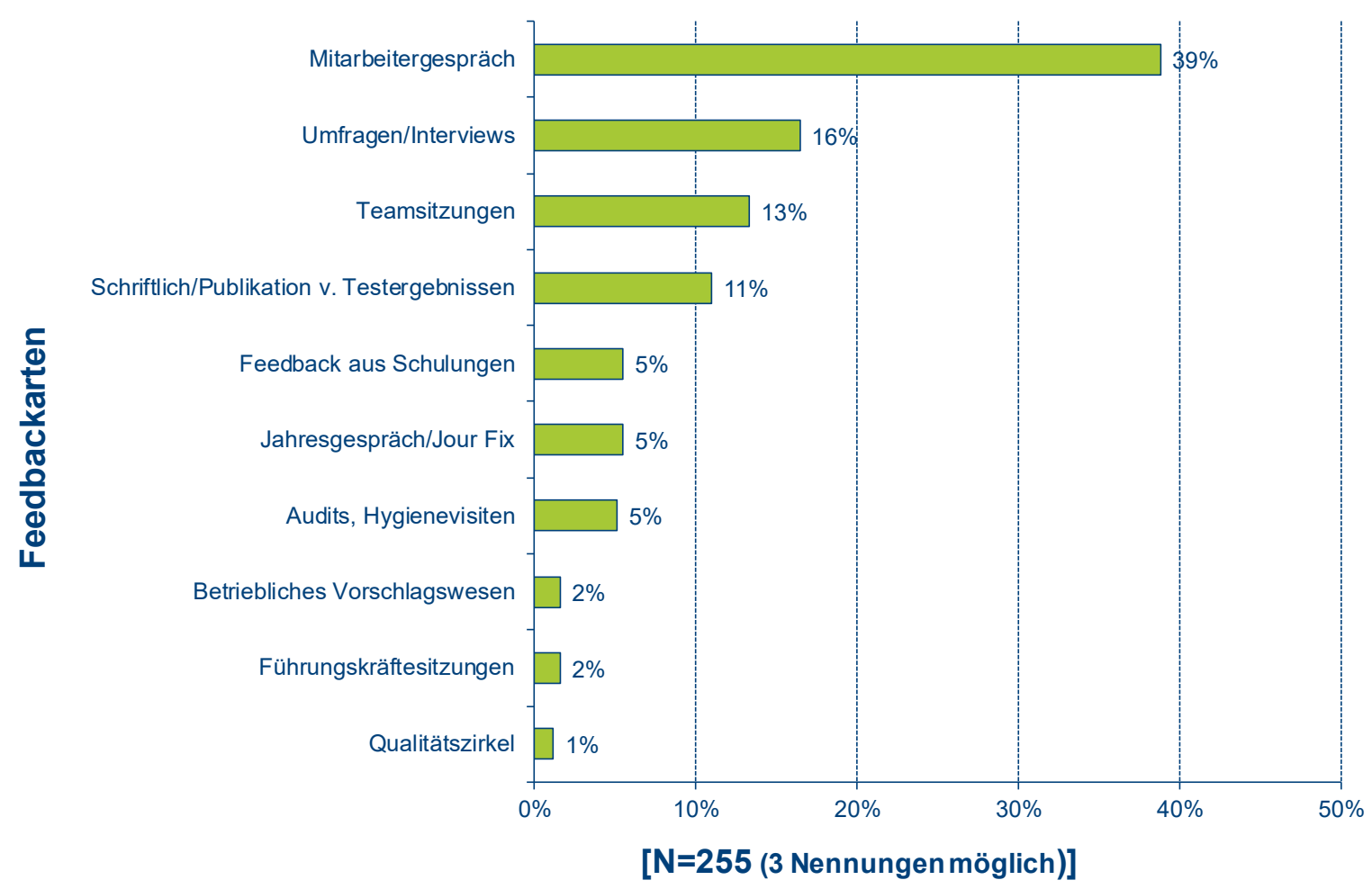

Abbildung 26: Feedbackarten 


\subsection{Einschätzung der Datensituation als Grundlage für ein Feed- back}

Hierbei hatten die Probanden die Möglichkeit, diese Frage mit sehr schlecht bis sehr gut zu bewerten. Der Umfrage lässt sich entnehmen, dass 2 Prozent dies als sehr schlecht einschätzen, 12 Prozent mit schlecht und die höchste Prozentzahl mit 44 Prozent bewertet dies weder mit schlecht, noch mit gut. 28 Prozent schätzen die Datensituation mit gut ein und nur 5 Prozent hingeben mit sehr gut.

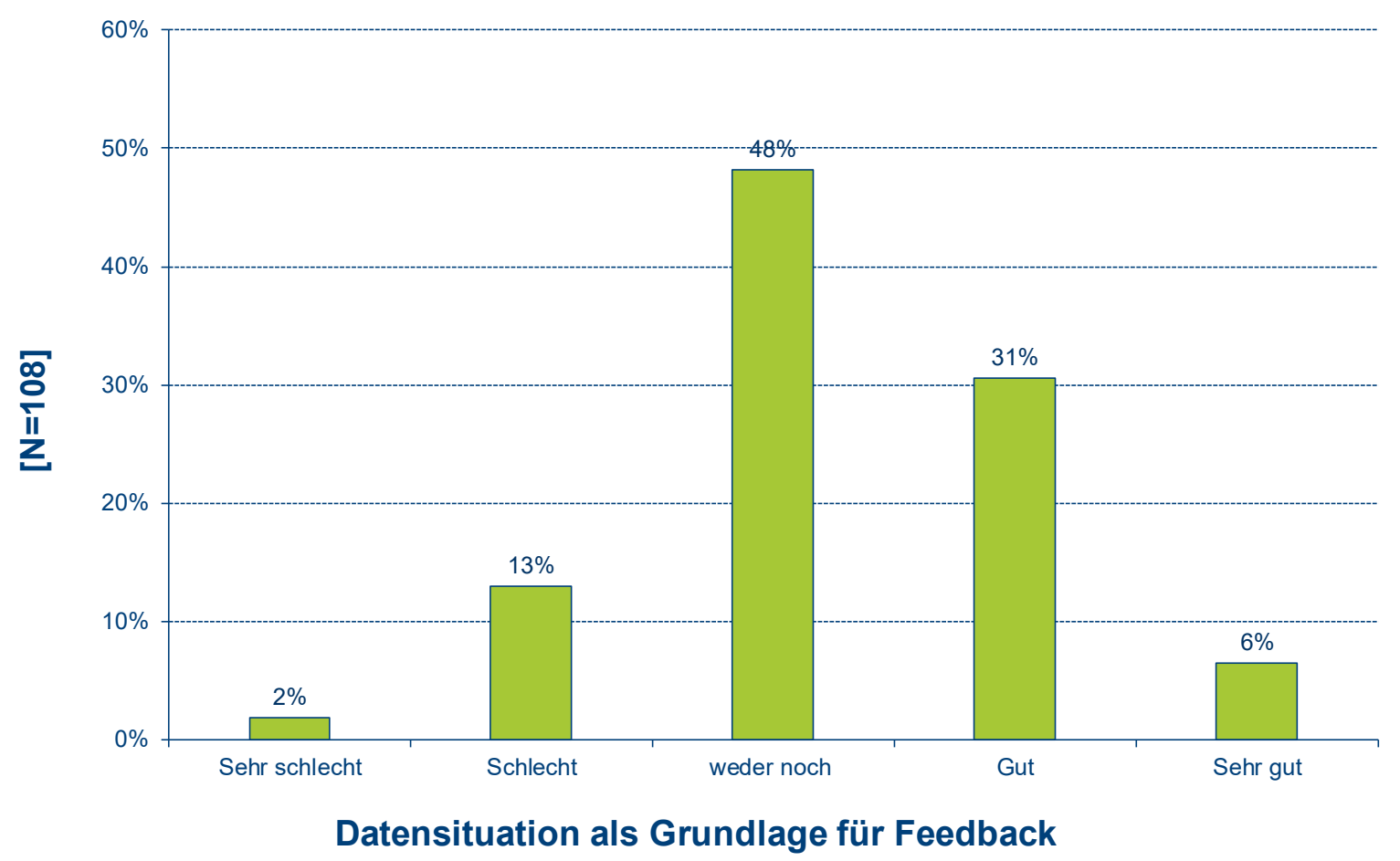

Abbildung 27: Einschätzung der Datensituation für Feedback 


\subsection{Angewandte Technologien}

Mit nur bis zu 5 Prozent wurden von den Befragten die Technologien Automatisiertes/zentrales Bestellsystem, Automatisierte Datenauswertung, wie zum Beispiel Hybase, Zählsysteme, Erinnerungen, wie beispielsweise Mails und Sonstiges, wie eine Stoppuhr, Funkchips oder akustische Signale bewertet. Sensoren, sowie die Visualisierung durch UV-Licht/Fluoreszenzmittel/Didaktobox wurden jeweils beide mit 7 Prozent bewertet und Observe durch z.B. Kameras, Tests wie ATP mit 13 Prozent. Die beiden am stärksten bewerteten Technologien sind mit 21 Prozent Schulungen, Aktionstage, Poster oder Videos und mit 40 Prozent Erfassungstools/Apps wie beispielsweise CleanHands oder NosoEx.

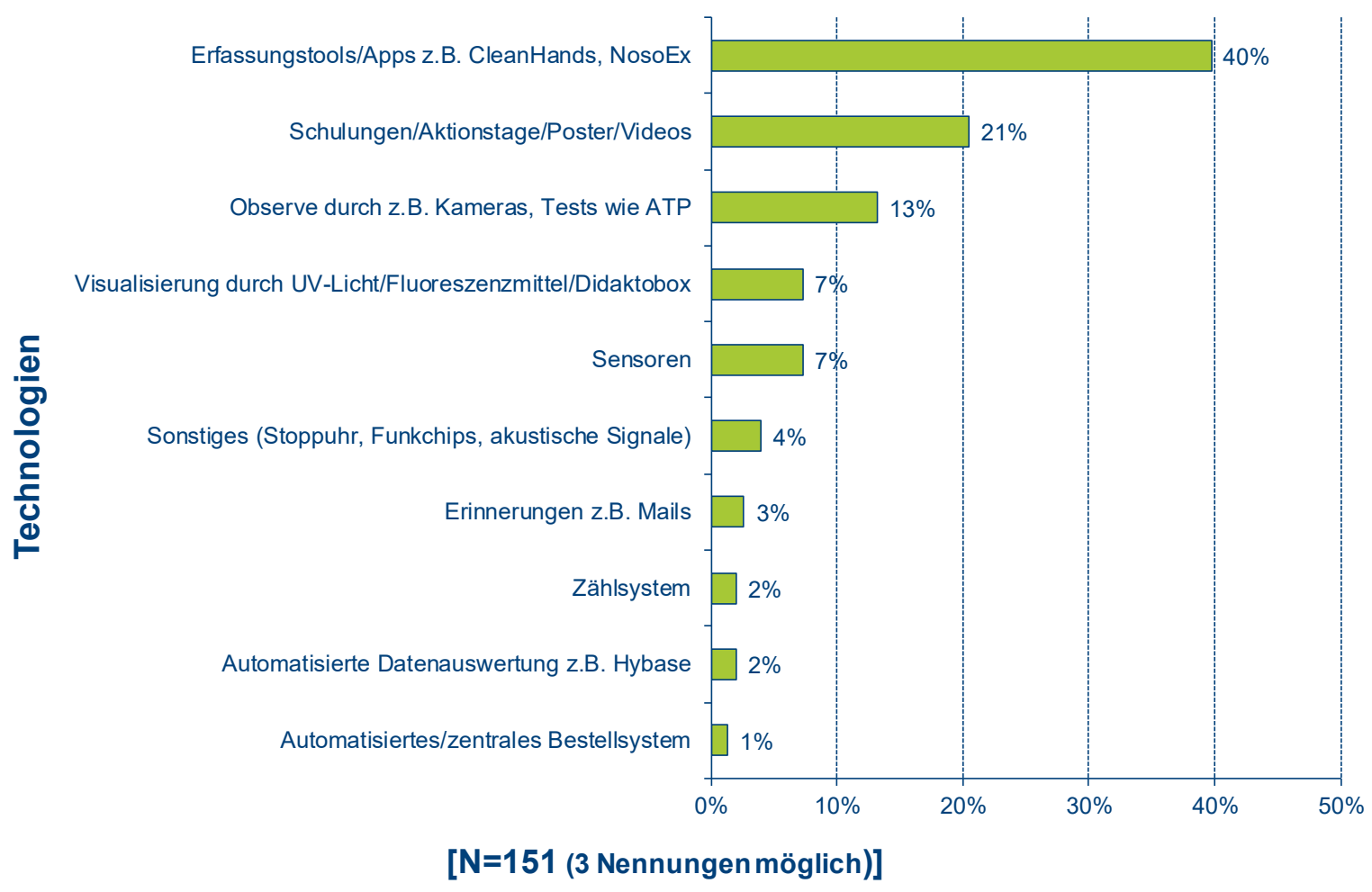

Abbildung 28: Technologien, die Compliance unterstützen 


\subsection{Unterstützung von Technologien in konkreten Aspekten}

40 Prozent der Probanden gaben an, dass die sich in der Erfassung und Auswertung durch Technologien Unterstützung erhoffen. Die Verbesserung von Compliance erhoffen sich 30 Prozent und 10 Prozent mehr Kontrolle. Im Bereich der Schulungen erhoffen sich 8 Prozent Unterstützung, sowie 6 Prozent im Bereich Awareness. 6 Prozent sind hingegen der Meinung, dass direkte Kommunikation hilfreicher ist.

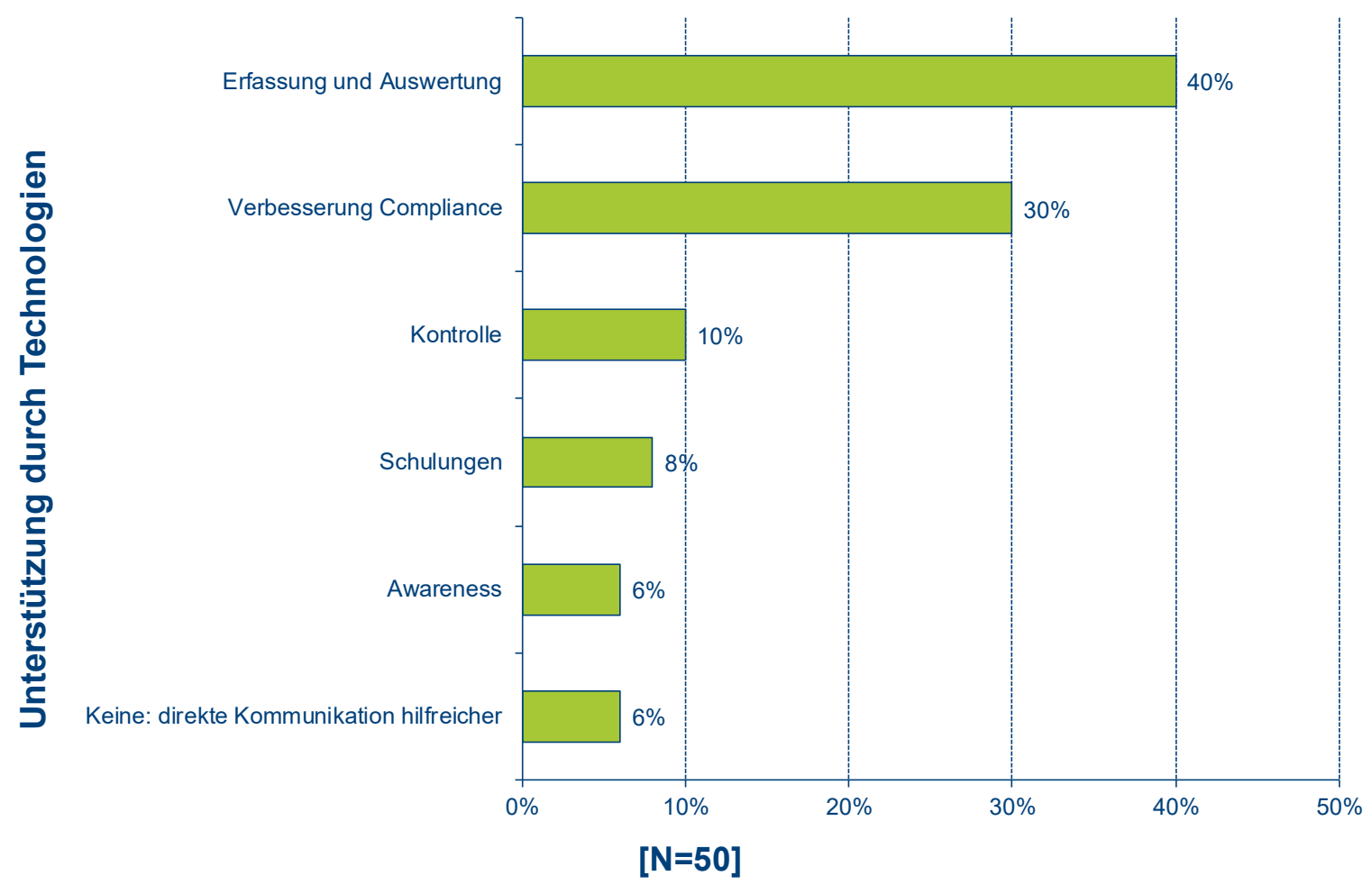

Abbildung 29: Bereiche, in denen Technologien unterstützen können 